Article

\title{
Pricing of Commodity Derivatives on Processes with Memory
}

\author{
Fred Espen Benth ${ }^{1, \dagger}$, Asma Khedher ${ }^{2, *, \dagger}$ and Michèle Vanmaele ${ }^{3,+} \mathbb{C}$ \\ 1 Department of Mathematics, University of Oslo, P.O. Box 1053, Blindern, N-0316 Oslo, Norway; \\ fredb@math.uio.no \\ 2 Korteweg-de Vries Institute for Mathematics, P.O. Box 94248, 1090 GE Amsterdam, The Netherlands \\ 3 Department of Applied Mathematics, Computer Science and Statistics, Ghent University, Krijgslaan 281 S9, \\ B-9000 Gent, Belgium; michele.vanmaele@ugent.be \\ * Correspondence: A.Khedher@uva.nl \\ + These authors contributed equally to this work.
}

Received: 31 October 2019; Accepted: 16 January 2020; Published: 21 January 2020

\begin{abstract}
Spot option prices, forwards and options on forwards relevant for the commodity markets are computed when the underlying process $S$ is modelled as an exponential of a process $\xi$ with memory as, e.g., a Volterra equation driven by a Lévy process. Moreover, the interest rate and a risk premium $\rho$ representing storage costs, illiquidity, convenience yield or insurance costs, are assumed to be stochastic. When the interest rate is deterministic and the risk premium is explicitly modelled as an Ornstein-Uhlenbeck type of dynamics with a mean level that depends on the same memory term as the commodity, the process $(\xi ; \rho)$ has an affine structure under the pricing measure $\mathbb{Q}$ and an explicit expression for the option price is derived in terms of the Fourier transform of the payoff function.
\end{abstract}

Keywords: equivalent measures; derivatives pricing; commodity markets; Langevin equation; affine processes; Fourier transform

\section{Introduction}

In financial markets, the arbitrage-free price of a derivative is derived by a risk neutral probability. In complete markets, the risk-neutral probability is unique, leading to a single arbitrage-free price dynamics reflecting the cost of perfect hedging. Most financial markets are, however, incomplete, with commodity markets as a typical case. Incompleteness can be a result of market frictions and non-Gaussianity in the price dynamics. One example of the former is the power spot market which is only accessible for physical players that can produce or transmit electricity. Other examples are oil and metals which are highly liquidly traded in many forward/futures markets. For such commodities, the spot market does not exist (although the front-month futures contract is sometimes referred to as a spot proxy). As in the interest rate theory, one models forward and futures price dynamics by referring to a spot dynamics which is non-existent in the market (analogous to short rates in the interest rate theory). Hence for these examples of commodities, we are in a highly incomplete market as the underlying spot cannot be traded. A further addition to incompleteness is that typically the dynamics in these markets cannot be expected to be Gaussian and thus non-Brownian processes are called for. See Benth et al. (2008); Eydeland and Wolyniec (2003); Geman (2005) for a discussion on pricing in energy and commodity markets, and Björk (2009) for a general treatment of the arbitrage pricing theory in financial markets.

There is no unique risk-neutral probability in an incomplete market. In the context of commodity markets with non-tradeable spots, any equivalent probability $\mathbb{Q}$ can serve as a measure for arbitrage-free pricing of derivatives like forwards and futures, and options on these. Following Benth et al. (2008), we refer to probabilities $\mathbb{Q} \sim \mathbb{P}$ as pricing measures. In this paper, we propose and 
study a class of probabilities $\mathbb{Q}$ that can be represented as a "deviation" from the risk-neutral one, in the sense that the price dynamics of the underlying asset will have a mean rate of return which can be represented as the sum of a risk-free interest rate and an additional yield under this probability $\mathbb{Q}$. With this class of probabilities, we model the risk premium by the additional yield. In commodity markets, this yield can be interpreted as storage costs, transportation, insurance, convenience yield and other illiquidity costs, see (Geman 2005, Ch. 2). Thus, the market price of risk is viewed as the compensation for financial risk and illiquidity risk. Our proposed class of pricing measures $\mathbb{Q}$ is going beyond this theory, being a mathematical representation in terms of an explicit change of probability and incorporating markets where one cannot resort to hedging arguments. Furthermore, the discounted spot price dynamics, which is a martingale process in the complete market situation, will now have an additional drift which is directly interpretable as the risk premium. Thus, our proposed class of pricing measures $\mathbb{Q}$ is benchmarked on the risk-neutral case, which is the natural reference point in this theory.

Important features in financial time series include fat tail distributions of risky asset returns, mean reversion, stationarity, and volatility clustering. See Cont (2001) for a discussion on the stylized empirical facts emerging from the statistical analysis of price variations in various types of financial markets. These features are well modelled by Volterra equations driven by Lévy processes and continuous-time autoregressive moving average (CARMA) processes making them popular modelling tools for power, gas, and oil prices (see Barndorff-Nielsen et al. (2013); Benth et al. (2014); Paschke and Prokopczuk (2010)) and weather variables like temperature and wind (see Benth and Benth (2012)), as well as volatility and turbulence (see Barndorff-Nielsen and Schmiegel (2009)).

In this paper, to model the logarithmic spot price dynamics, we consider a generalised Langevin equation which allows for dependency on the past in the current price, as well as jumps. The dependency on the past in the dynamics comes in as a memory term in the drift of the Langevin equation, being modelled as a weighted average of the historical logarithmic prices. The existence and uniqueness of a solution to the Langevin equation we consider in the paper follows from a result by Protter (1985).

Our first main result is that we give an expression for the unique solution of our proposed generalised Langevin equation. It turns out that this unique solution is of Volterra type with a memory in the diffusion and in the jumps. Our second main result is that we show that the class of pricing measures that we propose are indeed probabilities. Considering the generalised Langevin equation allows us to exploit the semimartingale theory in proving that the candidate density process in the Girsanov theorem is a true martingale. In particular, we appeal to the criteria in the extended Beneš method, developed in Klebaner and Liptser (2014). In our analysis we allow for stochastic interest rates and stochastic dynamics for the risk premium to which we impose a boundedness condition. This boundedness condition will allow our results on the measure change to hold. We specify models for the stochastic interest rates and for the risk premium to be jump-diffusions of Ornstein-Uhlenbeck type and we prove that they satisfy the imposed boundedness condition.

We perform an in-depth study of pricing of options and forwards using our pricing measure in the special cases when the logarithmic spot price dynamics is of Volterra type with memory in the diffusion and in the jumps. Pricing of options written on Lévy driven Volterra processes is also studied in Barndorff-Nielsen et al. (2013), where a different pricing measure is considered. Our approach allows to include stochastic interest rates and stochastic risk premium. Besides, it allows for a rather simple computation of the option prices. Indeed the dynamics of the discounted price process after the measure change that we propose is an affine SDE which does not include the memory term. Hence to compute option prices, we do not need the theory of affine Volterra equations as for example investigated in Abi Jaber et al. (2019) and Cuchiero and Teichmann (2018). We use the affine theory for regular SDEs and we obtain reasonably explicit expressions for call and put option prices. The forward price is also available explicitly as a function of the spot and the risk premium. Furthermore, we express the price dynamics for put and call options on forwards for this model class. Notice that 
plain vanilla European options are typically traded on forwards in many power markets. Further, we consider Wiener-driven continuous-time autoregressive moving average dynamics and extend the results in Benth and Benth (2012) to introduce a class of pricing measures $\mathbb{Q}$. For these models, we derive the forward price dynamics under our pricing measure. We remark that the explicit price expressions in all cases are derived under the assumption of deterministic interest rates and hence future and forward prices coincide.

Our analysis and results are presented as follows. Section 2 provides some motivation from commodity markets on pricing and presents the generalised Langevin equation that models the logarithmic spot prices. In Section 3, we prove the validity of our proposed measure change. Finally, Section 4 derives prices for various derivatives like options and forwards in the case of Volterra type of dynamics. An extensive analysis for the case of continuous-time autoregressive moving average processes is also included in this section.

\section{Generalised Langevin Equation}

Suppose that $\left(\Omega, \mathcal{F},\left(\mathcal{F}_{t}\right)_{0 \leq t \leq T}, \mathbb{P}\right)$ is a given probability space satisfying the usual conditions, see, e.g., Protter (2004), and $W=(W(t))_{0 \leq t \leq T}$ denotes an $\left(\mathcal{F}_{t}\right)$-Wiener process. Let $(E, \mathcal{E})$ be another measurable space. If the set $E$ is endowed with a metric, the Borel sigma algebra endowed by this metric will be denoted by $\mathcal{B}(E)$. Furthermore, we let $L=(L(t))_{0 \leq t \leq T}$ be an $\left(\mathcal{F}_{t}\right)$-Lévy process with characteristic triplet $\left(\varsigma, c^{2}, \ell\right)$. Assume that the Lévy measure of the process $L$ satisfies $\int z^{2} \ell(\mathrm{d} z)<$ $\infty$, e.g., $L$ is a square-integrable Lévy process. From the characteristic triplet of the process $L$, we know that the latter admits the following decomposition

$$
L(t)=b t+c W(t)+\int_{0}^{t} \int_{\mathbb{R}} z \tilde{N}(\mathrm{~d} s, \mathrm{~d} z), \quad t \in[0, T],
$$

where $b=\varsigma+\int_{|z| \geq 1} z \ell(\mathrm{d} z)$ and $\tilde{N}$ is a compensated Poisson random measure. That is $\tilde{N}(\mathrm{~d} t, \mathrm{~d} z)=$ $N(\mathrm{~d} t, \mathrm{~d} z)-\ell(\mathrm{d} z) \mathrm{d} t$ and $N(\mathrm{~d} t, \mathrm{~d} z)$ is the Poisson random measure such that $\mathbb{E}[N(\mathrm{~d} t, \mathrm{~d} z)]=\ell(\mathrm{d} z) \mathrm{d} t$. Denote by $\cdot{ }^{\top}$ the transpose of a given vector or a matrix.

In the next subsection, we introduce dynamics for the commodity spot market model.

\subsection{A Commodity Spot Market Model with Memory and Jumps}

Let $(S(t))_{0 \leq t \leq T}$ be our spot price dynamics in a commodity market and

$$
\xi(t):=\log S(t)
$$

with $(\xi(t))_{0 \leq t \leq T}$ being a generalised Langevin equation of the form

$$
\mathrm{d} \xi(t)=\left(\int_{0}^{t} M(t-u) \xi(u) \mathrm{d} u\right) \mathrm{d} t+\chi(t-) \mathrm{d} L(t),
$$

where $M$ is a measurable function on $\mathbb{R}_{+}$and $\chi$ is a strictly positive càdlàg deterministic function. The notation $\chi(t-)$ means $\lim _{s \uparrow t} \chi(s)$, i.e., the left-limit of the function. We assume the short rate $r$ is $\mathbb{F}$-adapted and locally Lebesgue integrable.

In principle we proposed a one-factor dynamics in (3) under the market probability $\mathbb{P}$. This model includes memory to take into account various existing spot price models such as Volterra equations driven by Lévy processes (see Section 4.1) as well as possible stochastic effects in the variance. There is a long-standing discussion whether commodities are stationary or not (discussed for example in Samuelson (2016) who insisted on stationarity for economic reasons). Based on empirical analysis in Barndorff-Nielsen et al. (2013), power spot prices in Germany are stationary, calling for a model with 
possible long-memory. However, forward and futures prices are typically not constant in the long end of the market, which should be a consequence of a stationary spot. Thus, one must either include a non-stationary term in the spot (which is what Schwartz and Smith (2000) do, also analysed in Paschke and Prokopczuk (2010)), or one can consider a measure change which turns the $\mathbb{P}$-dynamics of the spot into a non-stationary $\mathbb{Q}$-dynamics (see Ortiz-Latorre (2017)). We are following the latter stream of ideas, which are much more in line with existing models of the market dynamics, as well as the expected structure of the risk premium.

Denote by $\mathbb{D}_{[0, T]}$ the Skorohod space of càdlàg functions mapping $[0, T]$ to $\mathbb{R}$. Let $x_{[0, t)}=$ $\left\{x\left(t^{\prime}\right), t^{\prime}<t\right\}$. The SDE (3) under consideration belongs to the class of Volterra equations driven by a Lévy process. Those are SDEs of the type

$$
\xi(t)=\xi_{0}+\int_{0}^{t} a(s, \xi) \mathrm{d} s+\int_{0}^{t} b(s-, \xi) \mathrm{d} L(s)
$$

where $\xi_{0}$ is an $\mathcal{F}_{0}$-measurable random variable satisfying $\mathbb{P}\left(\left|\xi_{0}\right|<\infty\right)=1$ and $a(\cdot, \cdot)$ and $b(\cdot, \cdot)$ are $\mathcal{B}([0, T]) \times \mathcal{B}\left(\mathbb{D}_{[0, T]}\right)$-measurable functions of arguments $\left(s, x_{[0, s)}\right)$, for $s \in[0, T]$ and $x_{[0, s)} \in$ $\mathbb{D}_{[0, T]}$. Notice that in (4) and further on, we use for simplicity the shorthand notation $\xi$ for $\xi_{[0, s)}$ or $\xi_{[0, t)}$. The existence and uniqueness of the solution to the SDE of type (4) is well studied (see, e.g., (Liptser and Shiryayev 1984, Theorem 4.6) for Volterra equations driven by Brownian motion and Protter (1985) for Volterra equations driven by semimartingales). Using the results in this latter paper, we show in the following lemma, that under some integrability conditions on the kernel $M$ and its derivative, the SDE (3) has a unique solution.

Lemma 1. Assume that the kernel function $M$ is integrable over $[0, T]$. Then the SDE (3) admits a unique solution.

Proof. Notice that the SDE (3) is of type (4), with

$$
a(t, \xi)=\int_{0}^{t} M(t-s) \xi(s) \mathrm{d} s \quad \text { and } \quad b(t, \xi)=\chi(t) .
$$

Invoking Hölder's inequality, we obtain

$$
\begin{aligned}
\sup _{t \leq T}|a(t, \tilde{\xi})-a(t, \widetilde{\xi})| & =\sup _{t \leq T}\left|\int_{0}^{t} M(t-s)(\xi(s)-\tilde{\xi}(s)) \mathrm{d} s\right| \\
& \leq\left(\int_{0}^{T}|M(u)| \mathrm{d} u\right) \sup _{t \leq T}|\tilde{\xi}(t)-\widetilde{\xi}(t)| .
\end{aligned}
$$

Therefore $a$ satisfies a Lipschitz property given the integrability of $M$. Hence the result follows by invoking Theorem 4.3 in Protter (1985).

Volterra equations appear naturally in many areas of mathematics such as integral transforms, transport equations, and functional differential equations (we refer to Gripenberg et al. (1990) for an introduction and a general overview of these equations in the deterministic case). They also appear in applications in finance, see for example Abi Jaber et al. (2019); Cuchiero and Teichmann (2018); El Euch and Rosenbaum (2018); Gatheral et al. (2018); Holden et al. (2010). In the framework of stochastic delay equations and optimal control theory, we refer to Belbas (2007) and Øksendal and Zhang (2010). These processes have recently been proposed in the framework of modelling electricity and commodity prices, see, e.g., Barndorff-Nielsen et al. (2011) and Barndorff-Nielsen et al. (2014). 
When running a buy-and-hold strategy in a commodity market, the commodity must be stored. Thus the hedger will be incurred additional costs reflected in the forward price as an increased interest rate to be paid. On the other hand, holding the commodity has a certain advantage over being long a forward contract due to the greater flexibility. The notion of convenience yield is introduced to explain this additional benefit accrued to the owner of the physical commodity (see Eydeland and Wolyniec (2003); Geman (2005) for more details on convenience yield). To describe the added premium that the insurer charges to take on the risk, we introduce in the sequel the process $\rho$ which we refer to as the risk premium and which represents the increased interest rate or the convenience yield.

Empirical studies using returns from various indices and stocks indicate that the autocorrelation function of absolute returns and squared returns remains positive and decays slowly, remaining significantly positive over several days, sometimes weeks see, e.g., Comte and Renault (1996); Ding et al. (1993); Granger and Ding (1999). Equation (3) involving memory models well these features as it is shown for example in Takahashi (1996) and is relevant for many commodity markets including power (see, e.g., Barndorff-Nielsen et al. (2011) and Barndorff-Nielsen et al. (2014)). Later in Section 4, we will show that these types of models include Lévy driven Volterra equations which are popular models, e.g., for power, gas, and oil prices Barndorff-Nielsen et al. (2013); Benth et al. (2014); Paschke and Prokopczuk (2010).

\subsection{Analysis of the Langevin Equation}

Let $M$ be a function on $\mathbb{R}_{+}$. The Laplace transform of $M$ is given by

$$
\hat{M}(z)=\int_{0}^{+\infty} \mathrm{e}^{-z t} M(t) \mathrm{d} t
$$

for $z \in \mathbb{C}$, for which the integral makes sense. Let $H$ be defined through its Laplace transform by the relation

$$
\hat{H}(z)=\frac{H(0)}{z-\hat{M}(z)}
$$

where we assume $H(0)=1$. From (8), it follows that

$$
\dot{H}(t)=\int_{0}^{t} M(t-u) H(u) \mathrm{d} u,
$$

where $\dot{H}$ denotes the time derivative. The function $H$ is called the differential resolvent of $M$. Several properties of $H$ and its relation to solutions to Volterra integro-differential equations are discussed for example in (Gripenberg et al. 1990, Chapter 3).

As discussed in Lemma 1, we know that equation (3) has a unique solution under the integrability of the kernel $M$ over $[0, T]$. In the following proposition we provide an expression for the solution to (3). Notice that in our approach we do not impose the integrability of $M$. However we require the existence of a function $H$ such that (9) holds and the following assumption

Assumption 1. For any $T>0$, it holds

$$
\int_{0}^{T} \int_{0}^{t} \int_{0}^{u} M^{2}(t-u) H^{2}(u-s) \chi^{2}(s) \mathrm{d} s \mathrm{~d} u \mathrm{~d} t<\infty .
$$


Proposition 1. Consider the generalised Langevin Equation (3), which we recall to be

$$
\mathrm{d} \xi(t)=\int_{0}^{t} M(t-u) \xi(u) \mathrm{d} u \mathrm{~d} t+\chi(t-) \mathrm{d} L(t)
$$

where $\chi$ is a strictly positive, deterministic càdlàg function satisfying $\int_{0}^{T} \chi^{2}(s) \mathrm{d} s<\infty$. Assume there exists a unique solution $H$ to (9) such that Assumption 1 holds. Then

$$
\xi(t)=H(t) \xi(0)+\int_{0}^{t} H(t-u) \chi(u-) \mathrm{d} L(u)
$$

is the unique solution to the generalised Langevin equation (3).

Proof. The proof consists of two steps. First we show that $\xi(t)$ in (10) is indeed the unique solution to (3). In a second step we check that all necessary integrability conditions are satisfied.

Step 1. Computing the differential of $\xi(t)$ in (10), we get

$$
\mathrm{d} \xi(t)=\xi(0) \mathrm{d} H(t)+\chi(t-) \mathrm{d} L(t)+\int_{0}^{t} \frac{\mathrm{d}}{\mathrm{d} t} H(t-s) \chi(s-) \mathrm{d} L(s) \mathrm{d} t .
$$

For two solutions $\xi_{1}(t)$ and $\xi_{2}(t)$ of (11) with $\xi_{1}(0)=\xi_{2}(0)$, it immediately follows from (11) that $\xi_{1}(t)=\xi_{2}(t)$ for any $t \in[0, T]$. Thus (10) is the unique solution to (11). Now, we insert (9) in (11), perform a change of variables by putting $u=s+\tau$, and apply the stochastic Fubini theorem, see, e.g., Protter (2004), to arrive at

$$
\begin{aligned}
\mathrm{d} \xi(t)= & \int_{0}^{t} M(t-u) H(u) \mathrm{d} u \xi(0) \mathrm{d} t+\chi(t-) \mathrm{d} L(t) \\
& \quad+\int_{0}^{t} \int_{0}^{t-s} M(t-s-\tau) H(\tau) \mathrm{d} \tau \chi(s-) \mathrm{d} L(s) \mathrm{d} t \\
= & \int_{0}^{t} M(t-u) H(u) \mathrm{d} u \xi(0) \mathrm{d} t+\chi(t-) \mathrm{d} L(t) \\
& \quad+\int_{0}^{t} \int_{s}^{t} M(t-u) H(u-s) \mathrm{d} u \chi(s-) \mathrm{d} L(s) \mathrm{d} t \\
= & \left.\int_{0}^{t} M(t-u) H(u) \xi(0) \mathrm{d} u+\int_{0}^{t} M(t-u) H(u-s) 1_{\{s \leq u\}} \mathrm{d} u \chi(s-) \mathrm{d} L(s)\right] \mathrm{d} t \\
= & \int_{0}^{t} M(t-u)\left[\int_{0}^{t} M(t-u) H(u) \xi(0) \mathrm{d} u+\int_{0}^{t} M(t-u) \int_{0}^{u} H(u-s) \chi(s-) \mathrm{d} L(s) \mathrm{d} u\right] \mathrm{d} t \\
& \left.+\chi(t-) \mathrm{d} L(t) \quad \int_{0}^{t} H(u-s) \chi(s-) \mathrm{d} L(s)\right] \mathrm{d} u \mathrm{~d} t+\chi(t-) \mathrm{d} L(t) .
\end{aligned}
$$


By observing that the factor between brackets is exactly $\xi(u)$ according to (10), we conclude that

(11) and (3) are equivalent. Because (10) is the unique solution to (11) and $H$ satisfying (9) is unique, (10) is also the unique solution to (3).

Step 2. The differentiation under the stochastic integral in (11) follows by observing

$$
\begin{aligned}
\int_{0}^{t} \chi(u-) H(t-u) \mathrm{d} L(u) \\
=\int_{0}^{t} \chi(u-) \mathrm{d} L(u)+\int_{0}^{t} \chi(u-)(H(t-u)-H(0)) \mathrm{d} L(u) \\
=\int_{0}^{t} \chi(u-) \mathrm{d} L(u)+\int_{0}^{t} \int_{u}^{t} \chi(u-) \frac{\partial}{\partial s} H(s-u) \mathrm{d} s \mathrm{~d} L(u) \\
=\int_{0}^{t} \chi(u-) \mathrm{d} L(u)+\int_{0}^{t} \int_{0}^{s} \chi(u-) \frac{\partial}{\partial s} H(s-u) \mathrm{d} L(u) \mathrm{d} s .
\end{aligned}
$$

Using the stochastic Fubini theorem (Veraar 2012, Theorem 2.2 ), we deduce that a sufficient condition for the exchange of integrals in (13) is given by

$$
\int_{0}^{T} \int_{0}^{t} \chi^{2}(s)\left(\frac{\mathrm{d}}{\mathrm{d} t} H(t-s)\right)^{2} \mathrm{~d} s \mathrm{~d} t<\infty .
$$

Observe

$$
\begin{aligned}
\int_{0}^{t}\left(\frac{\mathrm{d}}{\mathrm{d} t} H(t-s)\right)^{2} \chi^{2}(s) \mathrm{d} s & =\int_{0}^{t}\left(\int_{0}^{t-s} M(t-s-u) H(u) \mathrm{d} u\right)^{2} \chi^{2}(s) \mathrm{d} s \\
& \leq T \int_{0}^{t} \int_{0}^{t-s} M^{2}(t-s-u) H^{2}(u) \chi^{2}(s) \mathrm{d} u \mathrm{~d} s \\
& =T \int_{0}^{t} \int_{0}^{u} M^{2}(t-u) H^{2}(u-s) \chi^{2}(s) \mathrm{d} s \mathrm{~d} u .
\end{aligned}
$$

Hence (14) follows from Assumption 1. To allow the exchange of the integrals in (12), we need to impose (see (Veraar 2012, Theorem 2.2 ))

$$
\int_{0}^{t} \int_{0}^{u} M^{2}(t-u) H^{2}(u-s) \chi^{2}(s) \mathrm{d} s \mathrm{~d} u<\infty,
$$

which again follows from Assumption 1.

The following proposition will allow us to investigate the link between our proposed generalised Langevin equation and CARMA processes in Section 4.

Proposition 2. Let $g$ be a real-valued function on $[0, \infty)$ and $H$ be a differentiable function such that

$$
g(t)=\dot{H}(t)
$$


where $H$ fulfils Assumption 1 and $H(0)=1$. Consider the $S D E$

$$
\mathrm{d} \xi(t)=\int_{0}^{t} g(t-u) \chi(u-) \mathrm{d} L(u) \mathrm{d} t+g(t) \xi(0) \mathrm{d} t+\chi(t-) \mathrm{d} L(t),
$$

where $\chi$ is a strictly positive, deterministic càdlàg function satisfying $\int_{0}^{T} \chi^{2}(s) \mathrm{d} s<\infty$. Then (17) admits a unique solution given by (10).

If there is a unique $M$ such that the relation (9) holds and $M$ and $H$ satisfy (15), then the SDE (17) is equivalent to the SDE (3).

Proof. Noticing that (17) is an SDE of type (11), the statement of the proposition follows immediately from the proof of Proposition 1.

SDEs of type (17) are common for modelling for example temperature and wind speed in energy markets, see Benth and Benth (2012). In Proposition 2, we showed the relation of these SDEs to the generalised Langevin equation (3). In Section 4.1, we will exploit this relation to show the existence of equivalent martingale measures for such processes.

The following remark concerns the extension of Propositions 1 and 2 to the multidimensional case.

Remark 1. Let $p \geq 1$ and $H, M:[0, T] \rightarrow \mathbb{R}^{p \times p}$ be matrix-valued functions. Assume $\operatorname{det}[z I-\hat{M}(z)] \neq 0$, for $z \in \mathbb{C}$. In this case the differential resolvent of $M$ translates to

$$
\hat{H}(z)=(z I-\hat{M}(z))^{-1},
$$

where I is the identity matrix. Assume $\chi$ is a $p$-dimensional vector with strictly positive components and $g$ as defined in (16) is a matrix-valued function, i.e., $g:[0, T] \rightarrow \mathbb{R}^{p \times p}$. Assumption 1 , with $M, H$ and $\chi$ adapted to the multidimensional case reads

$$
\int_{0}^{T} \int_{0}^{t} \int_{0}^{u}(M(t-u) H(u-s) \chi(s))^{\top}(M(t-u) H(u-s) \chi(s)) \mathrm{d} s \mathrm{~d} u \mathrm{~d} t<\infty .
$$

In this case, the results in Propositions 1 and 2 hold true and the solution $\xi$ will be a p-dimensional process.

To illustrate the construction of the function $H$ from a given memory function $M$, we consider the following example. This is a case where although $M$ is singular at zero, the corresponding function $H$ can be found.

Example 1. Let $M(s)=s^{-\alpha}$ for $0<\alpha<1 / 2$. Then we should find a function $H$ satisfying (9). This means, after integrating both sides and invoking Fubini,

$$
\begin{aligned}
H(t)-1=\int_{0}^{t} \dot{H}(s) \mathrm{d} s & =\int_{0}^{t} \int_{0}^{s}(s-u)^{-\alpha} H(u) \mathrm{d} u \mathrm{~d} s \\
& =\int_{0}^{t} \int_{u}^{t}(s-u)^{-\alpha} \mathrm{d} s H(u) \mathrm{d} u \\
& =\frac{1}{1-\alpha} \int_{0}^{t}(t-u)^{1-\alpha} H(u) \mathrm{d} u .
\end{aligned}
$$


To solve the Volterra integral equation, we propose that

$$
H(t)=\sum_{n=0}^{\infty} b_{n}(\alpha)\left(t^{2-\alpha}\right)^{n}
$$

Immediately, we find $b_{0}(\alpha)=1$. Inserting (20) into the integral equation (19), we get

$$
1+\frac{1}{1-\alpha} \sum_{n=0}^{\infty} b_{n}(\alpha) \int_{0}^{t}(t-s)^{1-\alpha} s^{2 n-n \alpha} \mathrm{d} s=\sum_{n=0} b_{n}(\alpha)\left(t^{2-\alpha}\right)^{n} .
$$

But the integral on the left hand side is connected to the Beta-distribution:

$$
\int_{0}^{t}(t-s)^{1-\alpha} s^{\beta} \mathrm{d} s=t^{2+\beta-\alpha} \int_{0}^{1}(1-u)^{1-\alpha} u^{\beta} \mathrm{d} u=t^{2+\beta-\alpha} \frac{\Gamma(2-\alpha) \Gamma(1+\beta)}{\Gamma(3+\beta-\alpha)} .
$$

With $\beta=2 n-n \alpha$ we find the recursive relations

$$
b_{n}(\alpha)=b_{n-1}(\alpha) \frac{\Gamma(2-\alpha) \Gamma(1+(n-1)(2-\alpha))}{(1-\alpha) \Gamma(1+n(2-\alpha))} .
$$

Using that $\Gamma(1+1-\alpha)=(1-\alpha) \Gamma(1-\alpha)$, we reach that $b_{0}(\alpha)=1$ and

$$
b_{n}(\alpha)=b_{n-1}(\alpha) \frac{\Gamma(1+(n-1)(2-\alpha))}{\Gamma(1+n(2-\alpha))} \Gamma(1-\alpha), n=1,2,3, \ldots
$$

Now, we may ask whether the representation (20) of $H(t)$ is a convergent series. By the ratio test we find

$$
\frac{b_{n}(\alpha)\left(t^{2-\alpha}\right)^{n}}{\left.b_{n-1}(\alpha)\right)\left(t^{2-\alpha}\right)^{n-1}}=\Gamma(1-\alpha) t^{2-\alpha} \frac{\Gamma(1+(n-1)(2-\alpha))}{\Gamma(1+n(2-\alpha))} .
$$

By Stirling's formula, we have an approximation of the Gamma-function for large values being

$$
\Gamma(1+x) \sim k \sqrt{x}(x / \mathrm{e})^{x}
$$

for some positive constant $k$. But then we have

$$
\begin{aligned}
\frac{\Gamma(1+(n-1)(2-\alpha))}{\Gamma(1+n(2-\alpha))} & \sim \sqrt{\frac{n-1}{n}} \frac{\left(\frac{(n-1)(2-\alpha)}{\mathrm{e}}\right)^{(n-1)(2-\alpha)}}{\left(\frac{n(2-\alpha)}{\mathrm{e}}\right)^{n(2-\alpha)}} \\
& =\sqrt{\frac{n-1}{n}}\left(\left(1-\frac{1}{n}\right)^{n} \frac{1}{n-1} \frac{\mathrm{e}}{2-\alpha}\right)^{2-\alpha} \rightarrow 0
\end{aligned}
$$

when $n \rightarrow \infty$, as $(n-1) / n \rightarrow 1,(1-1 / n)^{n} \rightarrow \mathrm{e}^{-1}$ and $0<\alpha<1 / 2$. Hence, $H$ is convergent for all $t<\infty$.

Two other examples of memory kernels with corresponding $H$ are discussed in Takahashi (1996). All three kernels are motivated as being particular types of influence functions that are consistent with the financial market. 


\section{Change of Measure}

Recall the spot market model introduced in Section 2.1, where we assume the conditions of Proposition 1 for the generalised Langevin dynamics (3).

We need the dynamics of $S$ under a pricing measure $\mathbb{Q}$ to price contingent claims on the commodity spot $S$. We study a particular class of pricing measures $\mathbb{Q}$ in terms of the stochastic process $\rho$ that should explain storage costs, illiquidity and/or convenience yield. We assume $\rho$ to be $\mathbb{F}$-adapted and locally square integrable with respect to the Lebesgue measure. We start by applying the Itô formula to $S=\exp (\xi)$ to derive the dynamics of $S$ under the market probability $\mathbb{P}$ from the dynamics of $\xi$ in (3)

$$
\begin{aligned}
\mathrm{d} S(t)=S(t) & \left(\int_{0}^{t} M(t-u) \log (S(u)) \mathrm{d} u+\chi(t) b+\gamma(t)+\frac{1}{2} \chi^{2}(t) c^{2}\right) \mathrm{d} t \\
& +S(t) \chi(t) c \mathrm{~d} W(t)+S(t-) \int_{\mathbb{R}}\left(\mathrm{e}^{\chi(t-) z}-1\right) \tilde{N}(\mathrm{~d} t, \mathrm{~d} z),
\end{aligned}
$$

where $\gamma(t)=\int_{\mathbb{R}}\left(\mathrm{e}^{\chi(t) z}-1-\chi(t) z\right) \ell(\mathrm{d} z)$. The dynamics of the discounted price process $\tilde{S}(t)=$ $\mathrm{e}^{-\int_{0}^{t} r(s) \mathrm{d} s} S(t), t \in[0, T]$, is given by

$$
\begin{aligned}
\mathrm{d} \tilde{S}(t)=\tilde{S}(t) & \left(\int_{0}^{t} M(t-u) \xi(u) \mathrm{d} u+\chi(t) b+\gamma(t)+\frac{1}{2} \chi^{2}(t) c^{2}-r(t)\right) \mathrm{d} t \\
& +\tilde{S}(t) \chi(t) c \mathrm{~d} W(t)+\tilde{S}(t-) \int_{\mathbb{R}}\left(\mathrm{e}^{\chi(t-) z}-1\right) \tilde{N}(\mathrm{~d} t, \mathrm{~d} z)
\end{aligned}
$$

We consider a pricing measure $\mathbb{Q}$ defined by $d \mathbb{Q}=Z(T) \mathrm{d} \mathbb{P}$ for a density process $(Z(t))_{0 \leq t \leq T}$ (see, e.g., the Girsanov Theorem 1.31 in Øksendal and Sulem (2009))

$$
\begin{aligned}
Z(t)= & \exp \left\{-\int_{0}^{t} \varphi(s, S) \mathrm{d} W(s)-\frac{1}{2} \int_{0}^{t} \varphi^{2}(s, S) \mathrm{d} s+\int_{0}^{t} \int_{\mathbb{R}} \log (1-\zeta(s-, z)) \tilde{N}(\mathrm{~d} s, \mathrm{~d} z)\right. \\
& \left.+\int_{0}^{t} \int_{\mathbb{R}}[\log (1-\zeta(s, z))+\zeta(s, z)] \ell(\mathrm{d} z) \mathrm{d} s\right\}, \quad 0 \leq t \leq T,
\end{aligned}
$$

where

$$
\begin{aligned}
& \varphi(t, S)=\frac{1}{\chi(t) c}\left(\int_{0}^{t} M(t-u) \log (S(u)) \mathrm{d} u+\chi(t) b+\frac{1}{2} \chi^{2}(t) c^{2}-r(t)-\rho(t)\right) \\
& \zeta(t, z)=\frac{\mathrm{e}^{\chi(t) z}-1-\chi(t) z}{\mathrm{e}^{\chi(t) z}-1}=1-\frac{\chi(t) z}{\mathrm{e}^{\chi(t) z}-1},
\end{aligned}
$$

with $(\rho(t))_{0 \leq t \leq T}$ being the risk premium process. When the measure $\mathbb{Q}$ exists, then

$$
\begin{aligned}
\mathrm{d} W_{\mathbb{Q}}(t) & =\varphi(t, S) \mathrm{d} t+\mathrm{d} W(t) \\
\tilde{N}_{\mathbb{Q}}(\mathrm{d} t, \mathrm{~d} z) & =\zeta(t, z) \ell(\mathrm{d} z) \mathrm{d} t+\tilde{N}(\mathrm{~d} t, \mathrm{~d} z)
\end{aligned}
$$


are respectively a Wiener process and a compensated jump measure under $\mathbb{Q}$ with compensator $\tilde{\ell}_{t}(\mathrm{~d} z) \mathrm{d} t=(1-\zeta(t, z)) \ell(\mathrm{d} z) \mathrm{d} t$. The dynamics of the discounted price process of $\tilde{S}$ under $\mathbb{Q}$ is

$$
\mathrm{d} \tilde{S}(t)=\tilde{S}(t-)\left(\chi(t) c \mathrm{~d} W_{\mathbb{Q}}(t)+\int_{\mathbb{R}}\left(\mathrm{e}^{\chi(t-) z}-1\right) \tilde{N}_{\mathbb{Q}}(\mathrm{d} t, \mathrm{~d} z)+\rho(t) \mathrm{d} t\right) .
$$

To prove the existence of the measure $\mathbb{Q}$, we need an additional assumption on the processes $r$ and $\rho$. Namely, $(r(t)+\rho(t))^{2}$ should be bounded by the maximum of $(\log S(t))^{2}$, for $t \in[0, T]$. Later we will specify models for $r$ and $\rho$ which are of Ornstein-Uhlenbeck type and which will satisfy the latter condition. Now we present the following result from (Klebaner and Liptser 2014, Theorem 5.1) which we will also need to prove the existence of the measure $\mathbb{Q}$.

Theorem 1. Let $a(\cdot, \cdot), b(\cdot, \cdot), \hat{\varphi}(\cdot, \cdot), h(\cdot, \cdot, \cdot)$, and $\zeta(\cdot, \cdot, \cdot)$ be measurable functions of arguments $\left(s, x_{[0, s)}\right)$ and $\left(s, x_{[0, s)}, z\right)$, for $s \in[0, T], x_{[0, s)} \in \mathbb{D}_{[0, T]}, z \in \mathbb{R}$. We assume that for any $x \in \mathbb{D}_{[0, T]}$, the functions $a(\cdot, x)$ and $b(\cdot, x)$ are square integrable with respect to $\mathrm{d} s$ on $[0, T]$, and for any $x \in \mathbb{D}_{[0, T]}$, the functions $h(\cdot, x, \cdot)$, and $\zeta(\cdot, x, \cdot)$ are square integrable with respect to $\ell(\mathrm{d} z) \mathrm{d}$ s on $\mathbb{R} \times[0, T]$. Furthermore we assume that $\zeta(s, x, z) \geq-1$, for all $s \in[0, T], x_{[0, s)} \in \mathbb{D}_{[0, T]}, z \in \mathbb{R}$. Let $X$ be given on $[0, T]$ by

$$
X(t)=X(0)+\int_{0}^{t} a(s, X) \mathrm{d} s+\int_{0}^{t} b(s, X) \mathrm{d} W(s)+\int_{0}^{t} \int_{\mathbb{R}} h(s-, X, z) \tilde{N}(\mathrm{~d} s, \mathrm{~d} z) .
$$

Define the martingale $\mathfrak{N}$ driven by $X$ and the functions $L$ and $\mathfrak{L}$ defined on $[0, T] \times \mathbb{D}_{[0, T]}$ as follows

$$
\begin{aligned}
\mathfrak{N}(t) & =\int_{0}^{t} \hat{\varphi}(s, X) \mathrm{d} W(s)+\int_{0}^{t} \int_{\mathbb{R}} \zeta(s-, X, z) \tilde{N}(\mathrm{~d} s, \mathrm{~d} z), \\
L(t, x)= & a^{2}(t, x)+b^{2}(t, x)+\int_{\mathbb{R}} h^{2}(t, x, z) \ell(\mathrm{d} z), \\
\mathfrak{L}(t, x)= & L(t, x)+b^{2}(t, x) \hat{\varphi}^{2}(t, x)+\int_{\mathbb{R}} h^{2}(t, x, z) \ell(\mathrm{d} z) \int_{\mathbb{R}} \zeta^{2}(t, x, z) \ell(\mathrm{d} z) \\
& +\int_{\mathbb{R}} h^{2}(t, x, z) \zeta(t, x, z) \ell(\mathrm{d} z) .
\end{aligned}
$$

Moreover, assume $|X(0)| \leq X_{0}$, where $X_{0}$ is a positive constant and for $x \in \mathbb{D}_{[0, T]}, t \in[0, T]$,

1. $\hat{\varphi}^{2}(t, x)+\int_{\mathbb{R}} \zeta^{2}(t, x, z) \ell(\mathrm{d} z) \leq X_{0}\left[1+\sup _{s<t} x^{2}(s)\right]$,

2. $L(t, x) \leq X_{0}\left[1+\sup _{s<t} x^{2}(s)\right]$,

3. $\mathfrak{L}(t, x) \leq X_{0}\left[1+\sup _{s<t} x^{2}(s)\right]$.

Then $z(t):=1+\int_{0}^{t} z(s-) \mathrm{d} \mathfrak{N}(s), 0 \leq t \leq T$, is a $\mathbb{P}$-martingale.

We state the existence of the pricing measure $\mathbb{Q}$ in the following proposition.

Proposition 3. Let $\xi$ be defined by (3), $\tilde{C}<\chi(t)<C$, for all $t \in[0, T]$, for strictly positive constants $\tilde{C}$ and $C$, and the initial condition $\xi(0)=\xi_{0}$ is bounded by a constant $\mathbb{P}$-a.s. Further, assume that the kernel function 
$M$ is integrable over $[0, T]$ and that there exists $H$ such that (9) holds. Moreover, let H satisfy Assumption 1 and the processes $r$ and $\rho$ satisfy

$$
(r(t)+\rho(t))^{2} \leq \hat{C}\left(1+\sup _{0 \leq s \leq t} \xi^{2}(s)\right), \quad 0 \leq t \leq T,
$$

for some positive constant $\hat{C}$ (possibly depending on $T$ ). Then, the process $(Z(t))_{0 \leq t \leq T}$ defined by (23) is a true $\mathbb{P}$-martingale and $\mathbb{E}[Z(T)]=1$.

Proof. As $S=\exp (\xi)$, the process $\varphi(24)$ can be denoted as a function of $t$ and $\xi$,

$$
\hat{\varphi}(t, \xi):=\varphi(t, S)=\frac{1}{\chi(t) c}\left[a(t, \xi)+\chi(t) b+\frac{1}{2} \chi^{2}(t) c^{2}-r(t)-\rho(t)\right],
$$

where the function $a(\cdot, \cdot)$ is given by (5). As by Proposition 1 the SDE (4) has a unique solution $\xi$, the result then follows by applying Theorem 1 with this semimartingale $\xi$ and the martingale given by

$$
\mathfrak{N}(t)=\int_{0}^{t} \hat{\varphi}(s, \xi) \mathrm{d} W(s)-\int_{0}^{t} \int_{\mathbb{R}} \zeta(s, z) \tilde{N}(\mathrm{~d} s, \mathrm{~d} z),
$$

with $\zeta(s, z)$ as defined in (25).

Hereto we prove that the conditions of Theorem 1 are satisfied.

- $\left|\xi_{0}\right|=|\eta|<C$ by the assumptions in this proposition.

- One can easily check that $\zeta(s, z)<1$, for all $s \in[0, T], z \in \mathbb{R}$.

- Using a similar approach as in the proof of Lemma 1 and the integrability of $M$ we obtain for $a(t, \xi)$ in (5)

$$
|a(t, \xi)|^{2} \leq C \sup _{0 \leq s \leq t}|\xi(s)|^{2}
$$

where $C$ is a positive constant. Next, the combination of assumption (27), Equations (28) and (29) and the uniform boundedness of $\chi$ leads to

$$
\begin{aligned}
\hat{\varphi}^{2}(t, \xi) & \leq \frac{3}{\chi^{2}(t) c^{2}}\left(a^{2}(t, \xi)+(r(t)+\rho(t))^{2}+\left[\chi(t) b+\frac{1}{2} \chi^{2}(t) c^{2}\right]^{2}\right) \\
& \leq C\left(1+\sup _{s \leq t} \xi^{2}(s)\right) .
\end{aligned}
$$

Let us turn our attention to $\zeta(t, z)$ : one can easily show that

$$
|\zeta(t, z)| \leq|\chi(t) z|
$$

Indeed consider for the moment the function

$$
f(u):=\frac{\mathrm{e}^{u}-1-u}{\mathrm{e}^{u}-1},
$$

for $u \in \mathbb{R}$. First, we observe that $\lim _{u \rightarrow 0} f(u)=0$, so the function does not have any singularity at zero. Consider next $u \geq 0$. Then, because $\exp (u)-1-u=\int_{0}^{u}(\exp (s)-1) \mathrm{d} s$, we find

$$
f(u)=\int_{0}^{u} \frac{\mathrm{e}^{s}-1}{\mathrm{e}^{u}-1} \mathrm{~d} s,
$$


which shows that $f(u) \geq 0$ for $u \geq 0$. Moreover, as $\exp (s)-1$ is an increasing function for $s \in[0, u]$, the integrand is less than 1 , and we find $f(u) \leq u$. For $u<0$, we consider the function

$$
g(v)=\frac{1-\mathrm{e}^{-v}-v}{1-\mathrm{e}^{-v}}, v>0,
$$

where we observe that $g(|u|)=f(u)$ for $u<0$. Then we find similar to above that $1-\exp (-v)-$ $v=-\int_{0}^{v}(1-\exp (-s)) \mathrm{d} s$. Hence,

$$
g(v)=-\int_{0}^{v} \frac{1-\mathrm{e}^{-s}}{1-\mathrm{e}^{-v}} \mathrm{~d} s
$$

Thus, we see that $g(v)<0$ and that the integrand is non-negative and less than 1 as $1-\exp (-s)$ is increasing for $s \in[0, v]$. It follows that $|g(v)| \leq v$ for $v>0$, or, $|f(u)| \leq|u|$. Letting $u:=\chi(t) z$, the desired inequality is reached. Hence, using again the uniform boundedness of $\chi$,

$$
\int_{\mathbb{R}} \zeta^{2}(t, z) \ell(\mathrm{d} z) \leq \int_{\mathbb{R}} \chi^{2}(t) z^{2} \ell(\mathrm{d} z) \leq \tilde{C} \int_{\mathbb{R}} z^{2} \ell(\mathrm{d} z)
$$

for a positive constant $\tilde{C}$. Adding up the latter and (30), we get

$$
\hat{\varphi}^{2}(t, \xi)+\int_{\mathbb{R}} \zeta^{2}(t, z) \ell(\mathrm{d} z) \leq K\left(1+\sup _{s \leq t} \xi^{2}(s)\right) .
$$

- Insertion of the decomposition (1) of the process $(L(t))_{0 \leq t \leq T}$ transforms the SDE (4) for $\xi$ into

$$
\mathrm{d} \xi(t)=(a(t, \xi)+\chi(t) b) \mathrm{d} t+\chi(t) c \mathrm{~d} W(t)+\chi(t-) \int_{\mathbb{R}} z \tilde{N}(\mathrm{~d} t, \mathrm{~d} z) .
$$

The bound (29) and the uniform boundedness of $\chi$, immediately provide

$$
L(t, \xi):=(a(t, \xi)+\chi(t) b)^{2}+\chi^{2}(t) c^{2}+\chi^{2}(t) \int_{\mathbb{R}} z^{2} \ell(\mathrm{d} z) \leq C\left(1+\sup _{s \leq t} \xi^{2}(s)\right) .
$$

- Noting that $\left(\mathrm{e}^{\chi(t) z}-1\right) \chi(t) z \geq 0$ implies $\zeta(t, z) \leq 1$, the uniform boundedness of $\chi$ and the estimates (31) and (32) lead to

$$
\begin{aligned}
\mathfrak{L}(t, \xi):= & L(t, \xi)+\chi^{2}(t) c^{2} \hat{\varphi}^{2}(t, \xi)+\chi^{2}(t) \int_{\mathbb{R}} z^{2} \ell(\mathrm{d} z) \int_{\mathbb{R}} \zeta^{2}(t, z) \ell(\mathrm{d} z) \\
& +\chi^{2}(t) \int_{\mathbb{R}} z^{2} \zeta(t, z) \ell(\mathrm{d} z) \\
\leq & C\left(1+\sup _{s \leq t} \xi^{2}(s)\right) .
\end{aligned}
$$

Hence the result follows.

Thus, we may conclude that under the assumptions of Proposition 3, a measure change exists and the memory in the drift of the dynamics of the asset price under the historical measure $\mathbb{P}$ does not enter the $\mathbb{Q}$-dynamics of the discounted price process (26). 
Remark 2. We emphasis once more that under $\mathbb{P}$ the spot price model has a memory in its dynamics, whereas under the pricing measure $\mathbb{Q}$ this effect is removed. However, the risk premium will inherit the memory.

Now, we discuss some explicit memory kernel $M$, interest rate $r$ and risk premium $\rho$ that satisfy the assumptions of Proposition 3. Recall the memory kernel $M$ singular at zero of Example 1 with which we can associate a differential resolvent $H$ as in (20) and (21). Because this latter $H$ is a continuous function in $t$, it is bounded for $t \in[0, T]$. Moreover, the function $\chi$ is assumed to be uniformly bounded in $t$ on $[0, T]$. Hence for Assumptions 1 to hold, it is enough to prove that

$$
\int_{0}^{T} \int_{0}^{t} M^{2}(t-u) \mathrm{d} u \mathrm{~d} t<\infty
$$

For $M(s)=s^{-\alpha}, \alpha>0$, we find that whenever $0<\alpha<1 / 2$

$$
\int_{0}^{T} \int_{0}^{t}(t-u)^{-2 \alpha} \mathrm{d} u \mathrm{~d} t=\frac{1}{1-2 \alpha} \int_{0}^{T} t^{1-2 \alpha} \mathrm{d} t=\frac{1}{(1-2 \alpha)(2-2 \alpha)} T^{2-2 \alpha}<\infty .
$$

Similarly, we find that $M$ is integrable over $[0, T]$ whenever $0<\alpha<1$ as

$$
\int_{0}^{T}|M(s)| \mathrm{d} s=\int_{0}^{T} s^{-\alpha} d s=\frac{1}{1-\alpha} T^{1-\alpha}<\infty .
$$

Note that a sufficient condition for both is the square integrability of this memory kernel $M$. Indeed,

$$
\max \left(\int_{0}^{T} \int_{0}^{t} M^{2}(t-u) \mathrm{d} u \mathrm{~d} t,\left(\int_{0}^{T}|M(s)| \mathrm{d} s\right)^{2}\right) \leq T \int_{0}^{T} M^{2}(s) \mathrm{d} s<\infty,
$$

with for $M(s)=s^{-\alpha}$, whenever $0<\alpha<1 / 2$,

$$
\int_{0}^{T} s^{-2 \alpha} d s=\frac{1}{1-2 \alpha} T^{1-2 \alpha}<\infty
$$

As dynamics for the processes $r$ and $\rho$ that satisfy assumption (27) we consider a bivariate Ornstein-Uhlenbeck (OU) type of dynamics with a mean level that depends on $\int_{0}^{t} M(t-u) \xi(u) \mathrm{d} u$. That is

$$
\begin{aligned}
\mathrm{d} r(t)=\left[A(t)-B_{2}(t) r(t)\right] \mathrm{d} t+B_{1}(t) \chi(t) c \mathrm{~d} W(t)+B_{1}(t) \chi(t-) \int_{\mathbb{R}} z \tilde{N}(\mathrm{~d} t, \mathrm{~d} z) \\
\begin{aligned}
\mathrm{d} \rho(t)=[ & \left.\bar{A}(t)+\bar{B}_{1}(t) \int_{0}^{t} M(t-u) \xi(u) \mathrm{d} u-\bar{B}_{2}(t) r(t)-\bar{B}_{3}(t) \rho(t)\right] \mathrm{d} t+\bar{B}_{1}(t) \chi(t) c \mathrm{~d} W(t) \\
& \quad+\bar{B}_{1}(t) \chi(t-) \int_{\mathbb{R}} z \tilde{N}(\mathrm{~d} t, \mathrm{~d} z) \\
r(0)= & r_{0} \in \mathbb{R}, \quad \rho(0)=\rho_{0} \in \mathbb{R}
\end{aligned}
\end{aligned}
$$

where $B_{i}, \bar{B}_{i}$, for $i \in\{1,2,3\}$ and $A, \bar{A}$ are deterministic functions uniformly bounded in $t$ by a constant.

The interest rate and the risk premium respectively in equations (33) and (34) are jump-diffusions of Ornstein-Uhlenbeck type driven by the same stochastic factors $W$ and $\tilde{N}$ as $\xi$. The dynamics in $\rho$ has a mean level that depends on the same memory as the commodity. These stochastic models are 
more general than a deterministic model and this particular choice is motivated by the aim to show that the estimate (27), that is crucial for the existence of the pricing measure, indeed holds. Moreover, when $r$ is deterministic, our choice for $\rho$ allows $(\xi, \rho)$ to have an affine structure and thus allows to derive explicit expressions for the options prices as it will be analysed in Section 4. An empirical study on the choice of the dynamics of $\rho$ is left for future research. However, notice that one can choose any stochastic dynamics for $\rho$ that allow the estimate (27) to hold or to choose for example the coefficients $\bar{B}_{i}, i=\{1,2,3\}$ in (34) to be very small.

To show the estimate (27), we apply in the proof of the following lemma, techniques similar to the proof of the Gronwall Inequality due to the particular Ornstein-Uhlenbeck-like structure of the dynamics of $r$ and $\rho$. The proof is detailed in Appendix B.

Lemma 2. Let $r, \rho$, and $\xi$ be respectively as in (33), (34), and (3) where we assume $A, \bar{A}, B_{i}, \bar{B}_{i}, i \in\{1,2,3\}$ to be uniformly bounded by a constant, $B_{1}$ and $\bar{B}_{1}$ to be functions of bounded variation on $[0, T], H(0)=1$, $\tilde{C}<\chi(t)<C$, for all $t \in[0, T]$, for strictly positive constants $\tilde{C}$ and $C$, and the initial condition $\xi(0)=\xi_{0}$ is bounded by a constant $\mathbb{P}$-a.s. Further, assume that the kernel function $M$ is integrable over $[0, T]$. Then the estimate (27) holds.

\section{Pricing of Options and Forwards}

In this section we derive analytical formulas for spot option prices and forwards when the underlying price process is modelled as $S=\exp (\xi)$, with $\xi$ satisfying (10) which is of interest in energy markets. We will focus on two types of processes depending on the SDE that model the dynamics of $\xi$. First, we consider an example where the process $\xi$ satisfies an SDE of the type (3) and the change of measure of Section 3 can be applied. For the second type, the process $\xi$ satisfies an SDE given by (17) and the results of Section 3 for the measure change cannot be applied. For this later case, an alternative argument will be developed.

\subsection{Example I: Volterra Equation Driven by a Lévy Process}

We consider a Volterra equation of convolution type of the form

$$
V(t)=\int_{0}^{t} H(t-s) \chi(s-) \mathrm{d} L(s),
$$

where $H$ is a real-valued function on $[0, \infty), \chi$ is a deterministic càdlàg positive function such that $\int_{0}^{T} \chi^{2}(s) \mathrm{d} s<\infty$, and $L$ is as in (1).

Assume that relation (9) holds for a unique $M$, and that Assumption 1 is satisfied. Then from Proposition 1, we know that (35) is the unique solution, with $V(0)=0$, to

$$
\mathrm{d} V(t)=\int_{0}^{t} M(t-s) V(s) \mathrm{d} s \mathrm{~d} t+\chi(t-) \mathrm{d} L(t)
$$

\subsubsection{Spot Option Prices}

In the following proposition we state the existence of a martingale measure to compute the option price written on $S=\exp (V)$. This result follows immediately from Proposition 3 and equation (36).

Proposition 4. Let the assumptions of Proposition 3 hold. Then we know there exists a martingale measure $\mathbb{Q}$ under which $\left(\mathrm{e}^{-\int_{0}^{t}(\rho(s)+r(s)) \mathrm{d} s} \mathrm{e}^{V(t)}\right)_{0 \leq t \leq T}$ is a $\mathbb{Q}$-martingale and the price of an (call/put) option written on $S$ is given by

$$
C(t)=\mathbb{E}^{\mathbb{Q}}\left[\mathrm{e}^{-\int_{t}^{T} r(s) \mathrm{d} s} \max (\varepsilon(S(T)-K), 0) \mid \mathcal{F}_{t}\right],
$$


for $t \leq T$, where $\varepsilon= \pm 1$ (for call and put, resp.). Here, $\mathbb{Q}$ is as described in (23) with $\varphi$ and $\zeta$, respectively as in (24) and (25).

Notice that the existence of an equivalent martingale measure for the spot price $S$ was proved by rewriting the process $V$ in terms of the kernel $M$, see (36). The proof would not be straightforward if using equation (35) directly.

Remark 3. As the memory in the spot dynamics is not explicitly present under the pricing measure $\mathbb{Q}$, the option price will not depend on this. On the other hand, as the option prices are functions of the spot price dynamics, the $\mathbb{P}$-dynamics of the options will inherit the memory effect. The situation is somewhat similar to the cointegration model analysed by Duan and Pliska (2004). They propose a two-dimensional stock price model with a common mean-reversion factor modelling the cointegration effect. After changing to the risk-neutral measure, the mean-reversion disappears and does not affect the pricing of derivatives (in their case, spread options).

When we assume $r$ to be a deterministic function of time and exploiting the affine structure under the measure $\mathbb{Q}$ of the process $(Z, \rho)$, we can derive an expression of the option price $C$ in terms of the Fourier transform of the payoff function. Hereto, we recalled in Appendix A some useful results on affine processes. In particular, we computed the moment generating function of affine processes in terms of the Fourier transform of the payoff function and solution to Riccati equations (see Theorem A1). For the sake of simplicity we put $\bar{B}_{1}=\bar{B}_{3}:=\bar{B}$ in the SDE for $\rho$ but the case $\bar{B}_{1} \neq \bar{B}_{3}$ can also be dealt with along similar lines leading to more involved expressions for the solutions of the Riccati equations.

Proposition 5. Let the assumptions of Proposition 3 hold. Assume $r$ is a deterministic function of time and that $r, \chi, \bar{A}, \bar{B}$, and $\bar{B}_{2}$ are continuous functions in $t \in[0, T]$. Define

$$
\iota(t, z)=\left[\begin{array}{ll}
\iota^{1}(t, z) & \iota^{2}(t, z)
\end{array}\right]^{\top}=\chi(t) z\left[\begin{array}{c}
1 \\
\bar{B}(t)
\end{array}\right],
$$

for $z \in \mathbb{R}$. Assume (A4) holds for $\iota(t, z)$ as defined in (38) and

$$
\int_{\{|z|>1\}} \frac{z}{\mathrm{e}^{\chi(t) z}-1} \mathrm{e}^{u_{1} \chi(t) z+\left[u_{2}+u_{1}(T-t)\right] \chi(t) z \bar{B}(t)} \ell(\mathrm{d} z)<\infty, \quad \forall t \in[0, T], \quad\left(u_{1}, u_{2}\right) \in \mathbb{C}^{2} .
$$

Define

$$
\tilde{f}(\lambda)=\frac{1}{2 \pi} \frac{K^{-(\omega-1+\mathrm{i} \lambda)}}{(\omega+\mathrm{i} \lambda)(\omega-1+\mathrm{i} \lambda)}, \quad \omega, \lambda \in \mathbb{R}
$$

and denote

$$
\begin{aligned}
& \omega_{1}(t)=r(t)-\frac{1}{2} \chi^{2}(t) c^{2}-\chi(t) \int_{\mathbb{R}} z\left(1-\frac{\chi(t) z}{\mathrm{e}^{\chi(t) z}-1}\right) \ell(\mathrm{d} z), \\
& \omega_{2}(t)=\bar{A}(t)+\bar{B}(t)\left(\omega_{1}(t)-\chi(t) b\right)-\bar{B}_{2}(t) r(t) .
\end{aligned}
$$

Then the price of an option written on $S$ is given by

$$
C(t)=\mathrm{e}^{-\int_{t}^{T} r(s) \mathrm{d} s} \int_{\mathbb{R}} \mathrm{e}^{\phi(t, T \omega+\mathrm{i} \lambda)+(T-t)(\omega+\mathrm{i} \lambda) \rho(t)} S^{\omega+i \lambda}(t) \tilde{f}(\lambda) \mathrm{d} \lambda,
$$


for

$$
\begin{cases}\omega>1, & \text { if } \varepsilon=1, \quad \text { call, } \\ \omega<0, & \text { if } \varepsilon=-1, \quad \text { put }\end{cases}
$$

where the function $\phi$ solves

$$
\begin{aligned}
& \partial_{t} \phi\left(t, u_{1}, u_{2}\right) \\
& =-\frac{1}{2} \chi^{2}(t) c^{2}\left[u_{1}+\bar{B}(t)\left(u_{2}+u_{1}(T-t)\right)\right]^{2}-\omega_{1}(t) u_{1}-\omega_{2}(t)\left(u_{2}+u_{1}(T-t)\right) \\
& -\int_{\mathbb{R}}\left(\exp \left\{\left[u_{1}+\bar{B}(t)\left(u_{2}+u_{1}(T-t)\right)\right] \chi(t) z\right\}-1\right. \\
& \left.\quad-\left[u_{1}+\bar{B}(t)\left(u_{2}+u_{1}(T-t)\right)\right] \chi(t) z\right) \frac{\chi(t) z}{\mathrm{e}^{\chi(t) z}-1} \ell(\mathrm{d} z) .
\end{aligned}
$$

Proof. Recall that $V=\log S$, and observe that the dynamics of $(V, \rho)$ under the measure $\mathbb{Q}$ is given by

$$
\begin{aligned}
\mathrm{d} V(t) & =\left(\rho(t)+\omega_{1}(t)\right) \mathrm{d} t+\chi(t) c \mathrm{~d} W_{\mathbb{Q}}(t)+\chi(t) \int_{\mathbb{R}} z \tilde{N}_{\mathbb{Q}}(\mathrm{d} t, \mathrm{~d} z), \\
\mathrm{d} \rho(t) & =\omega_{2}(t) \mathrm{d} t+\bar{B}(t) \chi(t) c \mathrm{~d} W_{\mathbb{Q}}(t)+\bar{B}(t) \chi(t) \int_{\mathbb{R}} z \tilde{N}_{\mathbb{Q}}(\mathrm{d} t, \mathrm{~d} z) .
\end{aligned}
$$

The parameters $\left(\varrho(t), \alpha(t), \omega(t), \beta(t), c, \gamma, \tilde{\ell}_{t}\right)$ as defined in (A1)-(A3) in Appendix A and associated with the processes $(V(t), \rho(t))$ and $R(t)=r(t)$ are given by

$$
\varrho(t)=\chi^{2}(t) c^{2}\left[\begin{array}{cc}
1 & \bar{B}(t) \\
\bar{B}(t) & \bar{B}^{2}(t)
\end{array}\right], \quad \alpha(t)=0, \quad \omega(t)=\left[\begin{array}{c}
\omega_{1}(t) \\
\omega_{2}(t)
\end{array}\right], \quad \beta(t)=\left[\begin{array}{ll}
0 & 1 \\
0 & 0
\end{array}\right],
$$

$c=0, \gamma=-1$, and $\tilde{\ell}_{t}(\mathrm{~d} z) \mathrm{d} t$ is the compensator of $N(\mathrm{~d} t, \mathrm{~d} z)$ under $\mathbb{Q}$. Hence by the assumptions in the proposition, the parameters $\left(\varrho(t), \alpha(t), \omega(t), \beta(t), c, \gamma, \tilde{\ell}_{t}\right)$ are strongly admissible parameters in the sense of Definition A1 in Appendix A. Let $(\phi, \psi)$ be a solution to the following Riccati equations

$$
\begin{aligned}
\partial_{t} \phi(t, T, u)=- & \frac{1}{2} \psi(t, T, u)^{\top} \varrho(t) \psi(t, T, u)-\omega^{\top}(t) \psi(t, T, u) \\
& -\int_{\mathbb{R}}\left(\mathrm{e}^{\psi(t, T, u)^{\top} \iota(t, z)}-1-\psi(t, T, u)^{\top} \iota(t, z)\right) \tilde{\ell}_{t}(\mathrm{~d} z), \\
\phi(T, T, u)= & 0, \\
\partial_{t} \psi(t, T, u)= & -\beta^{\top} \psi(t, T, u), \\
\psi(T, T, u)= & u,
\end{aligned}
$$

for $0 \leq t \leq T$ and $u \in \mathbb{C}^{2}$. Then there exists a unique global solution $(\phi(\cdot, T, u), \psi(\cdot, T, u)):[0, T] \rightarrow$ $\mathbb{C} \times \mathbb{C}^{2}$, for $u \in \mathbb{C}^{2}$ to the latter system given by

$$
\begin{aligned}
\phi(t, T, u)= & \int_{t}^{T} \frac{1}{2} \chi^{2}(s) c^{2}\left(\psi_{1}(s, T, u)+\bar{B}(s) \psi_{2}(s, T, u)\right)^{2} \mathrm{~d} s \\
& +\int_{t}^{T}\left(\omega_{1}(s) \psi_{1}(s, T, u)+\omega_{2}(s) \psi_{2}(s, T, u)\right) \mathrm{d} s \\
& +\int_{t}^{T} \int_{\mathbb{R}}\left(\exp \left\{\left[\psi_{1}(s, T, u)+\bar{B}(s) \psi_{2}(s, T, u)\right] \chi(s) z\right\}-1\right.
\end{aligned}
$$




$$
\begin{aligned}
& \left.\quad-\left[\psi_{1}(s, T, u)+\bar{B}(s) \psi_{2}(s, T, u)\right] \chi(s) z\right) \frac{\chi(s) z}{\mathrm{e}^{\chi(s) z}-1} \ell(\mathrm{d} z) \mathrm{d} s, \\
& \psi_{1}(t, T, u)=u_{1}, \\
& \psi_{2}(t, T, u)=u_{2}+u_{1}(T-t),
\end{aligned}
$$

where we used the fact that $\tilde{\ell}_{t}(\mathrm{~d} z)=(1-\zeta(t, z)) \ell(\mathrm{d} z)$, with $\zeta(t, z)$ given by (25). We fix $\omega>1$ and $u^{\top}=(\omega+\mathrm{i} \lambda, 0)$. The latter together with Theorem A1 and (Filipović 2009, Lemma 10.2) yield (37) for $\varepsilon=1$, where $\tilde{f}$ is as in (39). We fix $\omega<0$ and $u^{\top}=(\omega+\mathrm{i} \lambda, 0)$, then also (37) holds for $\varepsilon=-1$. Thus the statement of the proposition follows.

\subsubsection{Forward Prices}

We consider a forward contract on $S$, contracted at $t$ with time of delivery $T \geq t$ and with the forward price $F(t, T)$ such that

$$
\mathbb{E}^{\mathbb{Q}}\left[\mathrm{e}^{-\int_{t}^{T} r(s) \mathrm{d} s}(F(t, T)-S(T)) \mid \mathcal{F}_{t}\right]=0 .
$$

The latter is equivalent to

$$
F(t, T)=\left(\mathbb{E}^{\mathbb{Q}}\left[\mathrm{e}^{-\int_{t}^{T} r(s) \mathrm{d} s} \mid \mathcal{F}_{t}\right]\right)^{-1} \mathbb{E}^{\mathbb{Q}}\left[\mathrm{e}^{-\int_{t}^{T} r(s) \mathrm{d} s} S(T) \mid \mathcal{F}_{t}\right] .
$$

Assuming $r$ is deterministic, we state in the following proposition an analytical expression for the latter forward price (44).

Proposition 6. Let the assumptions of Proposition 3 hold. Moreover assume that $r$ is a deterministic function of time and that $r, \chi, \bar{A}, \bar{B}$, and $\bar{B}_{2}$ are continuous functions in $t \in[0, T]$ and $\iota^{2}(t, z)$ as defined in (38) satisfies the weak continuity conditions (A4). Moreover assume

$$
\int_{\{|z| \geq 1\}} \frac{z}{\mathrm{e}^{\chi(t) z}-1} \mathrm{e}^{\chi(t) z[1+(T-t) \bar{B}(t) \chi(t)]} \ell(\mathrm{d} z)<\infty, \quad \forall t \in[0, T] .
$$

Then the forward price (44) is given by

$$
F(t, T)=S(t) \exp \left(\mathcal{A}(t, T)+\int_{t}^{T} r(s) \mathrm{d} s+(T-t) \rho(t)\right)
$$

with

$$
\begin{aligned}
\mathcal{A}(t, T)= & \frac{1}{2} c^{2} \int_{t}^{T} \bar{B}^{2}(s) \chi^{2}(s)(T-s)^{2} \mathrm{~d} s+\int_{t}^{T}(T-s)\left[\bar{A}(s)+\left(\bar{B}(s)-\bar{B}_{2}(s)\right) r(s)\right] \mathrm{d} s \\
& -\left[b+\int_{\mathbb{R}} z \ell(\mathrm{d} z)\right] \int_{t}^{T} \bar{B}(s) \chi(s)(T-s) \mathrm{d} s-\frac{3}{2} c^{2} \int_{t}^{T} \bar{B}(s) \chi^{2}(s)(T-s) \mathrm{d} s \\
& +\int_{t}^{T} \int_{\mathbb{R}} \frac{\chi(s) z \mathrm{e}^{\chi(s) z}}{\mathrm{e}^{\chi(s) z}-1}\left[\mathrm{e}^{(T-s) \bar{B}(s) \chi(s) z}-1\right] \ell(\mathrm{d} z) \mathrm{d} s
\end{aligned}
$$

Proof. The process $\hat{Z}$ defined by

$$
\hat{Z}(t):=\frac{S(t)}{S(0)} \mathrm{e}^{-\int_{0}^{t}(r(s)+\rho(s)) \mathrm{d} s}, \quad 0 \leq t \leq T,
$$


is a positive $\mathbb{Q}$-martingale with $\mathbb{E}^{\mathbb{Q}}[\hat{Z}(t)]=1$ and defines a probability measure $\mathbb{Q} \sim \mathbb{Q}$ by

$$
\left.\frac{\mathrm{d} \hat{\mathbb{Q}}}{\mathrm{d} \mathbb{Q}}\right|_{\mathcal{F}_{t}}=\hat{Z}(t), \quad 0 \leq t \leq T .
$$

Considering a deterministic $r$ and applying Bayes' rule in (44), we get

$$
F(t, T)=S(t) \mathrm{e}^{\int_{t}^{T} r(s) \mathrm{d} s} \mathbb{E}^{\hat{\mathbb{Q}}}\left[\exp \left(\int_{t}^{T} \rho(s) \mathrm{d} s\right) \mid \mathcal{F}_{t}\right] .
$$

Moreover from the Girsanov theorem (see, e.g., (Øksendal and Sulem 2009, Theorem 1.31)), we know that

$$
\begin{aligned}
W_{\mathbb{Q}}(t) & =W_{\mathbb{Q}}(t)+\chi(t) c t \\
\tilde{N}_{\widehat{\mathbb{Q}}}(\mathrm{d} t, \mathrm{~d} z) & =\left(1-\mathrm{e}^{\chi(t) z}\right) \tilde{\ell}_{t}(\mathrm{~d} z) \mathrm{d} t+\tilde{N}_{\mathbb{Q}}(\mathrm{d} t, \mathrm{~d} z) .
\end{aligned}
$$

Recall the dynamics of $\rho$ under $\mathbb{Q}$ in (43). Then the dynamics of $\rho$ under $\hat{\mathbb{Q}}$ is given by

$$
\begin{aligned}
\mathrm{d} \rho(t)=\left[\omega_{2}(t)-\bar{B}(t) \chi^{2}(t) c^{2}-\bar{B}(t) \chi(t) \int_{\mathbb{R}} z\left(1-\mathrm{e}^{\chi(t) z}\right) \tilde{\ell}_{t}(\mathrm{~d} z)\right] \mathrm{d} t+\bar{B}(t) \chi(t) c \mathrm{~d} W_{\hat{\mathbb{Q}}}(t) \\
+\bar{B}(t) \chi(t) \int_{\mathbb{R}} z \tilde{N}_{\hat{\mathbb{Q}}}(\mathrm{d} t, \mathrm{~d} z) .
\end{aligned}
$$

Applying Theorem A1 in Appendix A, the expectation in (47) becomes

$$
\mathbb{E}^{\mathbb{Q}}\left[\exp \left(\int_{t}^{T} \rho(s) \mathrm{d} s\right) \mid \mathcal{F}_{t}\right]=\exp (\mathcal{A}(t, T)+\mathcal{B}(t, T) \rho(t)),
$$

where

$$
\begin{aligned}
\mathcal{B}(t, T)= & T-t \\
\mathcal{A}(t, T)= & \frac{1}{2} c^{2} \int_{t}^{T} \bar{B}^{2}(s) \chi^{2}(s)(T-s)^{2} \mathrm{~d} s-c^{2} \int_{t}^{T} \bar{B}(s) \chi^{2}(s)(T-s) \mathrm{d} s \\
& -\int_{t}^{T} \bar{B}(s) \chi(s)(T-s) \int_{\mathbb{R}} z\left(1-\mathrm{e}^{\chi(s) z}\right) \tilde{\ell}_{s}(\mathrm{~d} z) \mathrm{d} s+\int_{t}^{T} \omega_{2}(s)(T-s) \mathrm{d} s \\
& +\int_{t}^{T} \int_{\mathbb{R}}\left[\mathrm{e}^{(T-s) \bar{B}(s) \chi(s) z}-1-(T-s) \bar{B}(s) \chi(s) z\right] \hat{\ell}_{s}(\mathrm{~d} z) \mathrm{d} s,
\end{aligned}
$$

where $\hat{\ell}_{t}(\mathrm{~d} z) \mathrm{d} t=\mathrm{e}^{\chi(t) z} \tilde{\ell}_{t}(\mathrm{~d} z) \mathrm{d} t=\mathrm{e}^{\chi(t) z}(1-\zeta(t, z)) \ell(d z) \mathrm{d} t$ is the compensator of $N(\mathrm{~d} t, \mathrm{~d} z)$ under $\widehat{\mathbb{Q}}$. Substituting the expression for $\omega_{2}(t)$ from $(40)$ and $\zeta(t, z)$ from (25), combining and simplifying all these expressions in (47), we get the result.

\subsubsection{Options on Forwards}

In this subsection, we consider a call option with strike $K$ and exercise time $\tau \leq T$ written on the forward $F(\tau, T)$. Observe that the price at time $t \leq \tau \leq T$ of this option under the pricing measure $\mathbb{Q}$ defined by (23) will be

$$
P(t)=\mathbb{E}^{\mathbb{Q}}\left[\mathrm{e}^{-\int_{t}^{\tau} r(s) \mathrm{d} s}(F(\tau, T)-K)^{+} \mid \mathcal{F}_{t}\right],
$$

where $F$ is given by (45). 
In the following proposition, assuming that $r$ is deterministic, we derive an expression of the call option written on $F$ in terms of the Fourier transform of the payoff function.

Proposition 7. Let the assumptions of Proposition 6 hold. Define

$$
\begin{aligned}
\tilde{A}(t, T)= & -\frac{1}{2}(T-t)^{2} \bar{B}^{2}(t) \chi^{2}(t) c^{2}+(T-t) \bar{B}(t) \chi^{2}(t) c^{2}-\frac{1}{2} \chi^{2}(t) c^{2} \\
& +\int_{\mathbb{R}} \chi(t) z\left(\frac{\Sigma(t, T) z}{\mathrm{e}^{\chi(t) z}-1}-1\right) \ell(\mathrm{d} z)+\int_{\mathbb{R}} \frac{\chi(t) z \mathrm{e}^{\chi(t) z}}{\mathrm{e}^{\chi(t) z}-1}\left[\mathrm{e}^{(T-t) \bar{B}(t) \chi(t) z}-1\right] \ell(\mathrm{d} z), \\
\Sigma(t, T)= & (1+\bar{B}(t)(T-t)) \chi(t) .
\end{aligned}
$$

Assume the weak continuity conditions (A4) with $\iota(t, z)=\Sigma(t, T) z$. Recall the expression of $\tilde{f}$ in (39). Then price of the call option at time $t \leq \tau \leq T$ written on the forward $F$ and with maturity strike $K$ and maturity date $\tau$, is given by

$$
P(t)=\mathrm{e}^{-\int_{t}^{\tau} r(s) \mathrm{d} s} \int_{\mathbb{R}} \mathrm{e}^{\phi(\tau, T, \omega+\mathrm{i} \lambda)+(\omega+\mathrm{i} \lambda) \log F(\tau, T)} \tilde{f}(\lambda) \mathrm{d} \lambda, \quad \text { for } \omega>1,
$$

where the function $\phi$ solves

$$
\partial_{t} \phi(t, T, u)=-\frac{1}{2} c^{2} \Sigma^{2}(t, T) u^{2}-\tilde{A}(t, T) u-\int_{\mathbb{R}}\left(\mathrm{e}^{\Sigma(s, t) z u}-1-\Sigma(s, t) z u\right) \frac{\chi(t) z}{\mathrm{e}^{\chi(t) z}-1} \ell(\mathrm{d} z)
$$

and the process $(\log F(t, T))_{0 \leq t \leq T}$ satisfies the $S D E$

$$
\mathrm{d} \log F(t, T)=\tilde{A}(t, T) \mathrm{d} t+c \Sigma(t, T) \mathrm{d} W_{\mathbb{Q}}(t)+\Sigma(t, T) \int_{\mathbb{R}} z \tilde{N}_{\mathbb{Q}}(\mathrm{d} t, \mathrm{~d} z) .
$$

Proof. Recall that $S=\mathrm{e}^{V}$, and thus the expression (45) can be rewritten as

$$
F(t, T)=\mathrm{e}^{\mathrm{Y}(t, T)} \text { with } \mathrm{Y}(t, T)=V(t)+\mathcal{A}(t, T)+\int_{t}^{T} r(s) \mathrm{d} s+\rho(t)(T-t) .
$$

Taking the differential of $Y(t, T)$ and substituting the dynamics (42) and (43) of $V$ and $\rho$ under $\mathbb{Q}$ and the expression (46) for $\mathcal{A}(t, T)$ we get the dynamics (48) for $\log F(t, T)=\mathrm{Y}(t, T)$. Let $\phi$ and $\psi$ be a solution to the following Riccati equations

$$
\begin{aligned}
& \partial_{t} \phi(t, T, u)=- \frac{1}{2} c^{2} \Sigma^{2}(t, T) \psi^{2}(t, T, u)-\tilde{A}(t, T) \psi(t, T, u) \\
&-\int_{\mathbb{R}}\left(\mathrm{e}^{\psi(t, T, u) \Sigma(t, T) z}-1-\psi(t, T, u) \Sigma(t, T) z\right)(1-\zeta(t, z)) \ell(\mathrm{d} z), \\
& \phi(T, T, u)=0, \\
& \partial_{t} \psi(t, T, u)=0 \\
& \psi(T, T, u)=u,
\end{aligned}
$$

for $(t, u) \in \mathbb{R}_{+} \times \mathbb{C}$ and where $\zeta(t, z)$ is defined in (25). Then there exists a unique global solution $(\phi(\cdot, u), \psi(\cdot, u)): \mathbb{R}_{+} \rightarrow \mathbb{C} \times \mathbb{C}$, for all $u \in \mathbb{C}$ to the latter system given by

$$
\phi(t, T, u)=\int_{t}^{T}\left(\frac{1}{2} c^{2} \Sigma^{2}(s, T) \psi^{2}(s, T, u)+\tilde{A}(s, T) \psi(s, T, u)\right) \mathrm{d} s
$$




$$
\begin{aligned}
& +\int_{t}^{T} \int_{\mathbb{R}}\left(\mathrm{e}^{\psi(s, T, u) \Sigma(s, T) z}-1-\psi(s, T, u) \Sigma(s, T) z\right)(1-\zeta(t, z)) \ell(\mathrm{d} z) \mathrm{d} s, \\
\psi(t, T, u)= & u .
\end{aligned}
$$

We fix $\omega>1$ and $u=\omega+\mathrm{i} \lambda$. The latter together with Theorem A1 in Appendix A and (Filipović 2009, Lemma 10.2) yield the statement of the proposition.

Before we continue with the second example, we shortly discuss how one can estimate the model to data. First fit spot to spot price data and the short rate to interest rate data. When estimating the spot, one will encounter a model with memory as our initial model under $\mathbb{P}$. To gain information about the memory structure of the spot model, one can study the correlation structure using that model. Next include forward prices to assess the measure change and risk premium.

\subsection{Example II: CARMA Processes}

In this section we introduce a model that is used for modelling temperature and wind speed in weather derivatives, see (Benth and Benth 2012, §4). It is the continuous-time version of an ARMA model, namely a CARMA model where CARMA stands for continuous-time autoregressive moving average. For the special case of a CARMA $(2,1)$ model, one of the factors determining the temperature or wind speed satisfies an SDE of type (3). As noted in (Benth and Benth 2012, §6.2) stationary CARMA processes are special cases of Volterra equations driven by Brownian motions.

Let us introduce the model as in Benth and Benth (2012). Consider the stochastic vector process $\mathbf{X}$ taking values in $\mathbb{R}^{p}$, with $p \geq 1$, which is defined as the solution to the SDE

$$
\mathrm{d} \mathbf{X}(t)=A \mathbf{X}(t) \mathrm{d} t+\mathbf{e}_{p} \vartheta(t) \mathrm{d} W(t)
$$

where $\mathbf{e}_{k} \in \mathbb{R}^{p}, k=1, \ldots, p$, is the $k$ th standard Euclidean basis vector of $\mathbb{R}^{p}$. The $(p \times p)$-matrix $A$ is defined as

$$
\left[\begin{array}{ccccc}
0 & 1 & 0 & \cdots & 0 \\
0 & 0 & 1 & \cdots & 0 \\
\vdots & \vdots & \vdots & \ddots & \vdots \\
0 & 0 & 0 & \cdots & 1 \\
-\alpha_{p} & -\alpha_{p-1} & -\alpha_{p-2} & \cdots & -\alpha_{1}
\end{array}\right] .
$$

The constants $\alpha_{k}, k=1, \ldots, p$, are assumed to be non-negative with $\alpha_{p}>0$. The deterministic function $\vartheta: \mathbb{R}^{+} \rightarrow \mathbb{R}$ in the dynamics of $\mathbf{X}$ is assumed to be bounded, continuous and $\vartheta(t)$ is strictly bounded away from zero, i.e., there exists a constant $\bar{\vartheta}>0$ such that $\vartheta(t) \geq \bar{\vartheta}$ for all $t \geq 0$. Further, we will assume for the sake of simplicity that $\mathbf{X}(0)=\mathbf{0}$. As the entries in the matrix $A$ are constants it follows from (Liptser and Shiryayev 1984, Theorem 4.10) that the unique solution to the SDE (49) with initial condition $\mathbf{X}(0)=\mathbf{0}$ is given by

$$
\mathbf{X}(t)=\int_{0}^{t} \exp (A(t-s)) \mathbf{e}_{p} \vartheta(s) \mathrm{d} W(s) .
$$

This an SDE of type (10) considered in the multidimensional setting (see Remark 1) and with

$$
H(t)=\mathrm{e}^{A t}, \quad \chi(t)=\mathbf{e}_{p} \vartheta(t), \quad \mathbf{X}(0)=\mathbf{0} .
$$


From the existence of the solution (51), we infer that condition (14), adapted to this multidimensional setting holds, and thus we have

$$
\int_{0}^{T}\left(A \exp (A(t-s)) \mathbf{e}_{p}\right)^{\top} A \exp (A(t-s)) \mathbf{e}_{p} \vartheta^{2}(s) \mathrm{d} s<\infty .
$$

Then a similar computation as in Step 2 in the proof of Proposition 1 implies

$$
\mathrm{d} \mathbf{X}(t)=\mathbf{e}_{p} \vartheta(t) \mathrm{d} W(t)+\int_{0}^{t} A \mathrm{e}^{A(t-s)} \mathbf{e}_{p} \vartheta(s) \mathrm{d} W(s) \mathrm{d} t .
$$

The latter is an SDE of type (17) in the multidimensional setting with

$$
g(t)=A \mathrm{e}^{A t}, \quad \chi(t)=\mathbf{e}_{p} \vartheta(t), \quad \mathbf{X}(0)=\mathbf{0} .
$$

From relation (9), the kernel $M$ associated with the process $\mathbf{X}$ is such that

$$
g(t)=A \mathrm{e}^{A t}=\int_{0}^{t} M(t-u) H(u) \mathrm{d} u=\int_{0}^{t} M(t-u) \mathrm{e}^{A u} \mathrm{~d} u .
$$

A solution to the latter equation is given by $M(t)=A \delta_{0}(t)$, where $\delta_{0}$ is the Dirac delta function at zero. Using Proposition 1, we know $\mathbf{X}$ can be rewritten as

$$
\begin{aligned}
\mathrm{d} \mathbf{X}(t) & =\mathbf{e}_{p} \vartheta(t) \mathrm{d} W(t)+\int_{0}^{t} A \delta_{0}(t-u) \mathbf{X}(u) \mathrm{d} u \mathrm{~d} t \\
& =\mathbf{e}_{p} \vartheta(t) \mathrm{d} W(t)+A \mathbf{X}(t) \mathrm{d} t,
\end{aligned}
$$

and we recover equation (49) for $\mathbf{X}$. Of course, in this particular case this latter result follows straightforwardly from (53). Here we wanted to point out that the process $\mathbf{X}$ fits into our framework.

For $0 \leq q<p$, define the vector $\mathbf{b} \in \mathbb{R}^{p}$ with coefficients $b_{j}, j=0,1, \ldots, p-1$ satisfying $b_{q}=1$ and $b_{j}=0$ for $q<j<p$. We define the $\operatorname{CARMA}(p, q)$ process $Y$ as

$$
Y(t)=\mathbf{b}^{\top} \mathbf{X}(t) .
$$

Substituting the expression (51) for $\mathbf{X}(t)$, we can express $Y$ as

$$
Y(t)=\int_{0}^{t} \mathbf{b}^{\top} \exp (A(t-s)) \mathbf{e}_{p} \vartheta(s) \mathrm{d} W(s) .
$$

It is evident that $Y$ is a Gaussian process with mean zero and variance

$$
\int_{0}^{t}\left(\mathbf{b}^{\top} \exp (A(t-s)) \mathbf{e}_{p}\right)^{2} \vartheta^{2}(s) \mathrm{d} s .
$$

There are two special cases of the $\operatorname{CARMA}(p, q)$. First, when $\mathbf{b}=\mathbf{e}_{1}$, then $q=0$ and we obtain a so-called CAR $(p)$ process that is used to model temperature. Second, requiring $q=p-1$ then we get for example for $p=2$, a CARMA $(2,1)$ model that can be used to model wind speed. 
Applying the Itô formula to (56), we find as dynamics of the process $Y$

$$
\mathrm{d} Y(t)=\left(\int_{0}^{t} \mathbf{b}^{\top} A \exp (A(t-s)) \mathbf{e}_{p} \vartheta(s) \mathrm{d} W(s)\right) \mathrm{d} t+\mathbf{b}^{\top} \mathbf{e}_{p} \vartheta(t) \mathrm{d} W(t) .
$$

Clearly, the drift term involves the whole path of the Wiener process up to time $t \geq 0$.

The SDE (57) for $Y$ is also of type (17) with

$$
g(t)=\mathbf{b}^{\top} A \exp (A t) \mathbf{e}_{p}, \quad H(t)=\mathbf{b}^{\top} \exp (A t) \mathbf{e}_{p}, \quad \chi(t)=\vartheta(t), \quad Y(0)=0 .
$$

In order to apply the measure change proposed in Section 3, we need that the drift in (57) can be written as $\int_{0}^{t} M(t-s) Y(s) \mathrm{d} s$ for some memory function $M$. Substituting (51) in (57) we have

$$
\mathrm{d} Y(t)=\mathbf{b}^{\top} A \mathbf{X}(t) \mathrm{d} t+\mathbf{b}^{\top} \mathbf{e}_{p} \vartheta(t) \mathrm{d} W(t),
$$

and hence $M$ must satisfy the equation

$$
\mathbf{b}^{\top} A \mathbf{X}(t)=\int_{0}^{t} M(t-s) Y(s) \mathrm{d} s .
$$

We emphasize that $M$ is a scalar function here. Because $Y(t)=\mathbf{b}^{\top} \mathbf{X}(t)$, we find

$$
\mathbf{b}^{\top} A \mathbf{X}(t)=\mathbf{b}^{\top} \int_{0}^{t} M(t-s) \mathbf{X}(s) \mathrm{d} s .
$$

Take the Laplace transform on both sides to find

$$
\mathbf{b}^{\top} A \hat{\mathbf{X}}(\theta)=\mathbf{b}^{\top}(\hat{M}(\theta) \hat{\mathbf{X}}(\theta)),
$$

for all $\theta \in \mathbb{R}$, where $\hat{M}(\theta)$ is the Laplace transform (7) of $M$ and $\hat{\mathbf{X}}(\theta)$ is a vector in $\mathbb{R}^{p}$ consisting of the Laplace transform of the coordinates of $\mathbf{X}$. But then we see that $\hat{M}(\theta)$ is an eigenvalue of $A$ for all $\theta \in \mathbb{R}$ with eigenvector $\hat{\mathbf{X}}(\theta)$. As $A$ has fixed eigenvectors and eigenvalues, this cannot be true and we conclude that there exists no $M$ such that (58) holds.

Hence, to show the existence of a measure change for a process being the exponential of a CARMA, we will give an alternative argument. We will extend the result in (Benth and Benth 2012, Proposition 5.1) to study the pricing in our context. To this end, for a CARMA $(p, q)$-process $Y(t)=\mathbf{b}^{\top} \mathbf{X}(t)$, define the spot dynamics as

$$
S(t)=\exp (\mu t+Y(t)),
$$

where we suppose that $\mathbf{X} \in \mathbb{R}^{p}$ follows the Ornstein-Uhlenbeck dynamics with level $\xi \in \mathbb{R}^{p}$,

$$
\mathrm{d} \mathbf{X}(t)=(\boldsymbol{\xi}+A \mathbf{X}(t)) \mathrm{d} t+\vartheta \mathbf{e}_{p} \mathrm{~d} W(t) .
$$

A simple application of the multidimensional Itô formula gives the $\mathbb{P}$-dynamics of $S$ as

$$
\mathrm{d} S(t)=\left(\mu+\frac{1}{2}\left(\mathbf{b}^{\top} \mathbf{e}_{p}\right)^{2} \vartheta^{2}+\mathbf{b}^{\top}(\boldsymbol{\xi}+A \mathbf{X}(t))\right) S(t) \mathrm{d} t+\vartheta\left(\mathbf{b}^{\top} \mathbf{e}_{p}\right) S(t) \mathrm{d} W(t) .
$$

For simplicity we suppose that $\vartheta$ is constant.

We recall that $\mathbf{b}^{\top}=\left(b_{0}, b_{1}, \ldots, b_{q}, 0 \ldots, 0\right) \in \mathbb{R}^{p}$, where $q<p$ and $b_{q}=1$. If $q<p-1$, then $\mathbf{b}^{\top} \mathbf{e}_{p}=0$ because the last coordinate of $\mathbf{b}$ in this case is zero. Hence, if $q<p-1$ we do not have 
any martingale term in the dynamics of $S$ and therefore cannot change measure to any $\mathbb{Q} \sim \mathbb{P}$ so that $\exp (-r t) S(t)$ is a $\mathbb{Q}$-martingale. We assume from now on that

$$
q=p-1
$$

In this case, $\mathbf{b}^{\top} \mathbf{e}_{p}=1$ and the dynamics of $S$ under $\mathbb{P}$ is

$$
\mathrm{d} S(t)=\left(\mu+\frac{1}{2} \vartheta^{2}+\mathbf{b}^{\top}(\boldsymbol{\xi}+A \mathbf{X}(t))\right) S(t) \mathrm{d} t+\vartheta S(t) \mathrm{d} W(t)
$$

Introduce next a state-dependent risk premium,

$$
\rho(t):=\mathbf{c}^{\top}(\boldsymbol{\theta}+C \mathbf{X}(t)),
$$

where $\boldsymbol{\theta} \in \mathbb{R}^{p}, \mathbf{c}^{\top}=\left(c_{0}, c_{1}, \ldots, c_{\tilde{q}}, 0, \ldots, 0\right) \in \mathbb{R}^{p}, \tilde{q}<p$ and

$$
C=\left[\begin{array}{ccccc}
0 & 1 & 0 & \cdots & 0 \\
0 & 0 & 1 & \cdots & 0 \\
\vdots & \vdots & \vdots & \ddots & \vdots \\
0 & 0 & 0 & \cdots & 1 \\
-\beta_{p} & -\beta_{p-1} & -\beta_{p-2} & \cdots & -\beta_{1}
\end{array}\right]
$$

for $\beta_{i}>0, i=1, \ldots, p$. The natural choice of "moving-average vector" $\mathbf{c}$ is, as we will see, $\mathbf{c}=\mathbf{b}$.

Define the stochastic process

$$
\theta(t, \mathbf{X})=\vartheta^{-1}\left(\mu+\frac{1}{2} \vartheta^{2}-r+\mathbf{b}^{\top}(\boldsymbol{\xi}+A \mathbf{X}(t))-\rho(t)\right) .
$$

With the specification of the risk premium as in (62), we find

$$
\theta(t, \mathbf{X})=\frac{\mu+\frac{1}{2} \vartheta^{2}-r+\mathbf{b}^{\top} \xi-\mathbf{c}^{\top} \boldsymbol{\theta}}{\vartheta}+\vartheta^{-1}\left(\mathbf{b}^{\top} A-\mathbf{c}^{\top} C\right) \mathbf{X}(t) .
$$

Hence, $\theta$ is a constant plus a linear combination of the coordinates of $\mathbf{X}(t)$.

In the following proposition we state the existence of a martingale measure to compute the option price written on $S$ as given in (61).

Proposition 8. Let $S$ be as in (61) with $\boldsymbol{X}$ defined by the SDE (49) and let $q=p-1$. Assume that the eigenvalues of the matrix $A$ all have negative real part, the risk-free interest rate $r$ is constant and $\rho$ is defined by (62). Then there exists a martingale measure $\mathbb{Q}$ under which $\left(\mathrm{e}^{-\int_{0}^{t} \rho(s) \mathrm{d} s-r t} S(t)\right)_{0 \leq t \leq T}$ is a $\mathbb{Q}$-martingale and the price of an (call/put) option written on $S$ is given by

$$
C(t)=\mathrm{e}^{-r(T-t)} \mathbb{E}^{\mathbb{Q}}\left[\max (\varepsilon(S(T)-K), 0) \mid \mathcal{F}_{t}\right],
$$

for $t \leq T$ and $\varepsilon= \pm 1$ (call and put, resp.), where $\mathbb{Q}$ is given by

$$
\left.\frac{\mathrm{d} \mathbb{Q}}{\mathrm{d} \mathbb{P}}\right|_{\mathcal{F}_{t}}=Z(t)=\exp \left[-\int_{0}^{t} \theta(s, \boldsymbol{X}) \mathrm{d} W(s)-\frac{1}{2} \int_{0}^{t} \theta^{2}(s, \boldsymbol{X}) \mathrm{d} s\right], \quad t \in[0, T],
$$

$\theta(t, \boldsymbol{X})$ is as in (65), and the $\mathbb{Q}$-dynamics of $S$ is given by

$$
\mathrm{d} S(t)=(r+\rho(t)) S(t) \mathrm{d} t+\vartheta S(t) \mathrm{d} W_{\mathbb{Q}}(t),
$$


for a $\mathbb{Q}$-Brownian motion $W_{\mathbb{Q}}$.

Proof. The result follows immediately by extending the result of (Benth and Benth 2012, Proposition 5.1 $)^{1}$ to the case of a non-zero mean level $\xi$ where the measure change as described in this proposition takes the form of

$$
\theta_{0}(s)=\mu+\frac{1}{2} \vartheta^{2}-r+\mathbf{b}^{\top} \boldsymbol{\xi}-\mathbf{c}^{\top} \boldsymbol{\theta}, \quad \theta^{\prime}=\mathbf{b}^{\top} A-\mathbf{c}^{\top} C, \quad \sigma(s)=\vartheta .
$$

Note that for the measure change to be structure preserving the components $\theta_{i}$ in the vector $\theta^{\prime}$ should satisfy

$$
\theta_{i}<\alpha_{i}, i=1, \ldots, p,
$$

as it is assumed in (Benth and Benth 2012, Proposition 5.1).

By definition, $\mathbf{X}(t)$ is a $p$-variate Ornstein-Uhlenbeck process, and thus $\mathbf{X}$ is a $p$-variate Gaussian process with mean and variance (under $\mathbb{P}$ ) being bounded as long as $A$ has eigenvalues with negative real part.

Let us next analyse the $\mathbb{Q}$-dynamics of $\mathbf{X}$ for the choice of $\theta$ given in (65). We find,

$$
\begin{aligned}
\mathrm{d} \mathbf{X}(t)=( & \left.\xi-\mathbf{e}_{p}\left(\mu+\frac{1}{2} \vartheta^{2}-r\right)-\mathbf{e}_{p}\left(\mathbf{b}^{\top} \boldsymbol{\xi}-\mathbf{c}^{\top} \boldsymbol{\theta}\right)\right) \mathrm{d} t \\
& +\left(A \mathbf{X}(t)-\mathbf{e}_{p}\left(\mathbf{b}^{\top} A-\mathbf{c}^{\top} C\right) \mathbf{X}(t)\right) \mathrm{d} t+\vartheta \mathbf{e}_{p} \mathrm{~d} W_{\mathbb{Q}}(t) .
\end{aligned}
$$

Observe that $\mathbf{e}_{p}\left(\mathbf{b}^{\top} A \mathbf{X}(t)\right)=\left(\mathbf{e}_{p} \mathbf{b}^{\top}\right) A \mathbf{X}(t)$. Hence, the second drift term in the $\mathbb{Q}$-dynamics of $\mathbf{X}(t)$ above is therefore equal to $\left(A-\left(\mathbf{e}_{p} \mathbf{b}^{\top}\right) A+\left(\mathbf{e}_{p} \mathbf{c}^{\top}\right) C\right) \mathbf{X}(t)$. But

$$
\mathbf{e}_{p} \mathbf{b}^{\top}=\left[\begin{array}{ccccc}
0 & 0 & 0 & \cdots & 0 \\
0 & 0 & 0 & \cdots & 0 \\
\vdots & \vdots & \vdots & \ddots & \vdots \\
0 & 0 & 0 & \cdots & 0 \\
b_{0} & b_{1} & b_{2} & \cdots & b_{p-1}
\end{array}\right]
$$

where we recall $b_{p-1}=1$. It follows,

$$
\left(\mathbf{e}_{p} \mathbf{b}^{\top}\right) A=\left[\begin{array}{ccccc}
0 & 0 & 0 & \cdots & 0 \\
0 & 0 & 0 & \cdots & 0 \\
\vdots & \vdots & \vdots & \ddots & \vdots \\
0 & 0 & 0 & \cdots & 0 \\
-\alpha_{p} & b_{0}-\alpha_{p-1} & b_{1}-\alpha_{p-2} & \cdots & b_{p-2}-\alpha_{1}
\end{array}\right]
$$

1 The proof of this extension requires a reasoning using the spectral representation applied to the function $g_{\xi}(u)=\theta^{\prime} \exp (A u) \xi$ similarly as applied to the function $g(u)=\theta^{\prime} \exp (A u) \mathbf{e}_{p}$ in the proof of (Benth and Benth 2012, Proposition 5.1). 
Similarly, for $\tilde{q}=p-1$,

$$
\left(\mathbf{e}_{p} \mathbf{c}^{\top}\right) C=\left[\begin{array}{ccccc}
0 & 0 & 0 & \cdots & 0 \\
0 & 0 & 0 & \cdots & 0 \\
\vdots & \vdots & \vdots & \ddots & \vdots \\
0 & 0 & 0 & \cdots & 0 \\
-c_{p-1} \beta_{p} & c_{0}-c_{p-1} \beta_{p-1} & c_{1}-c_{p-1} \beta_{p-2} & \cdots & c_{p-2}-c_{p-1} \beta_{1}
\end{array}\right]
$$

We conclude that

$$
\begin{aligned}
A & =\left(\mathbf{e}_{p} \mathbf{b}^{\top}\right) A+\left(\mathbf{e}_{p} \mathbf{c}^{\top}\right) C \\
& =\left[\begin{array}{ccccc}
0 & 1 & 0 & \cdots & 0 \\
0 & 0 & 1 & \cdots & 0 \\
\vdots & \vdots & \vdots & \ddots & \vdots \\
0 & 0 & 0 & \cdots & 1 \\
-c_{p-1} \beta_{p} & -b_{0}+c_{0}-c_{p-1} \beta_{p-1} & -b_{1}+c_{1}-c_{p-1} \beta_{p-2} & \cdots & -b_{p-2}+c_{p-2}-c_{p-1} \beta_{1}
\end{array}\right] .
\end{aligned}
$$

Choosing $\mathbf{c}=\mathbf{b}$ yields that $A-\left(\mathbf{e}_{p} \mathbf{b}^{\top}\right) A+\left(\mathbf{e}_{p} \mathbf{c}^{\top}\right) C=C$. Therefore,

$$
\mathrm{d} \mathbf{X}(t)=(\widetilde{\boldsymbol{\xi}}+C \mathbf{X}(t)) \mathrm{d} t+\vartheta \mathbf{e}_{p} \mathrm{~d} W_{\mathbb{Q}}(t),
$$

where

$$
\widetilde{\xi}=\boldsymbol{\xi}-\mathbf{e}_{p}\left(\mu+\frac{1}{2} \vartheta^{2}-r\right)+\mathbf{e}_{p} \mathbf{b}^{\top}(\boldsymbol{\theta}-\boldsymbol{\xi}) .
$$

Observe that $\widetilde{\xi}$ only changes in the last coordinate compared to $\xi$. In conclusion, we have that $\mathbf{X}$ has $A$ as "speed of mean-reversion" under $\mathbb{P}$, while $C$ under $\mathbb{Q}$.

Proposition 9. Let $S$ be as in (61) with $X$ defined by the SDE (49) and let $q=p-1$. Assume that the eigenvalues of the matrix $A$ all have negative real part, the risk-free interest rate $r$ is constant and $\rho$ is defined by (62) with $\boldsymbol{c}=\boldsymbol{b}$. Then the forward price (44) of the forward contract written on $S$ is given by

$$
\begin{aligned}
F(t, T)= & \exp \left(\mu T+\mathbf{b}^{\top} \exp (C(T-t)) \mathbf{X}(t)+\mathbf{b}^{\top}(\exp (C(T-t))-I) C^{-1} \tilde{\xi}\right) \\
& \times \exp \left(\frac{\vartheta^{2}}{2} \int_{0}^{T-t}\left(\mathbf{b}^{\top} \exp (C s) \mathbf{e}_{p}\right)^{2} \mathrm{~d} s\right)
\end{aligned}
$$

for $t \leq T$ and with $C$ the matrix (63) and I the $p \times p$ identity matrix.

Proof. Recalling that $S(T)$ equals $\exp \left(\mu T+\mathbf{b}^{\top} \mathbf{X}(T)\right)$ according to (59), with

$$
\mathbf{X}(T)=\exp (C(T-t)) \mathbf{X}(t)+(\exp (C(T-t))-I) C^{-1} \tilde{\boldsymbol{\xi}}+\vartheta \int_{t}^{T} \exp (C(T-s)) \mathbf{e}_{p} \mathrm{~d} W_{\mathbb{Q}}(s)
$$

as solution to (67), the forward price can be expressed as

$$
\begin{aligned}
F(t, T)= & \mathbb{E}^{\mathbb{Q}}\left[S(T) \mid \mathcal{F}_{t}\right] \\
= & \exp \left(\mu T+\mathbf{b}^{\top} \exp (C(T-t)) \mathbf{X}(t)+\mathbf{b}^{\top}(\exp (C(T-t))-I) C^{-1} \tilde{\xi}\right) \\
& \quad \times \mathbb{E}^{\mathbb{Q}}\left[\exp \left(\vartheta \int_{t}^{T} \mathbf{b}^{\top} \exp (C(T-s)) \mathbf{e}_{p} \mathrm{~d} W_{\mathbb{Q}}(s)\right)\right] .
\end{aligned}
$$


Evaluating the mean of a lognormal random variable combined with the isometry property provides the stated result.

Note that solving the SDE (66) for $S$ leads to

$$
F(t, T)=\mathbb{E}^{\mathbb{Q}}\left[S(T) \mid \mathcal{F}_{t}\right]=\mathrm{e}^{r(T-t)} S(t) \mathbb{E}^{\mathbb{Q}}\left[\exp \left(\int_{t}^{T} \rho(s) \mathrm{d} s+\vartheta\left(W_{\mathbb{Q}}(T)-W_{\mathbb{Q}}(t)\right)\right) \mid \mathcal{F}_{t}\right],
$$

where $\rho(s)=\mathbf{b}^{\top}(\boldsymbol{\theta}+C \mathbf{X}(s))$ depends on the process $\mathbf{X}$ that satisfies the SDE (67). This approach however will not lead easily to the forward price (69).

Author Contributions: All three authors contributed equally to the conceptualization, formal analysis and the writing of the original draft of this paper. All authors have read and agree to the published version of the manuscript.

Funding: The research by Fred Espen Benth was funded by the project FINEWSTOCH of the Research Council of Norway with grant number 239019.

Acknowledgments: The authors acknowledge the Centre of Advanced Study (CAS) at the Norwegian Royal Academy of Science and Letters (Program SEFE) for providing occasions of research discussions to start this paper. Michèle Vanmaele acknowledges the Research Foundation Flanders (FWO) and the Special Research Fund (BOF) of the Ghent University for providing the possibility to go on sabbatical leave to CAS. The authors thank Fabian Andsem Harang for some discussions. Two anonymous referees and an external editor are thanked for their careful reading and critics, improving the presentation of the paper.

Conflicts of Interest: The authors declare no conflict of interest.

\section{Appendix A. Affine Processes}

As affine processes play an important role in our considerations, we include in this Appendix some useful results on this class of processes.

Affine processes are continuous-time Markov processes characterised by the fact that their $\log$-characteristic function depends in an affine way on the initial state vector of the process. Recently affine models have gained significant attention in the finance literature mainly due to their analytical tractability (see for example Duffie et al. (2003), Duffie et al. (2000), and Keller-Ressel and Mayerhofer (2015)). The process $(\xi, r, \rho)$ introduced in (3)-(34) is a time-inhomogeneous affine process under the measure $\mathbb{Q}$. As we are interested in pricing contingent claims written on a process $\exp (\xi)$, we recall in this subsection a result showing that pricing contingent claims in time-inhomogeneous affine models can be reduced to the solution of a set of Riccati-type ordinary differential equations.

Denote by Sem ${ }^{d}$ the convex cone of symmetric positive semidefinite $d \times d$ matrices. Assume $X$ is an $\mathbb{R}^{d}$-valued process with dynamics given by the following stochastic differential equation (SDE)

$$
\begin{aligned}
\mathrm{d} X(t) & =\omega(t, X(t)) \mathrm{d} t+\sigma(t, X(t)) \mathrm{d} \tilde{W}(t)+\int_{\mathbb{R}} \iota(t, z)\left(\mu^{X}(\mathrm{~d} t, \mathrm{~d} z)-v(\mathrm{~d} t, \mathrm{~d} z)\right), \\
X(0) & =x \in \mathbb{R}^{d},
\end{aligned}
$$

where $\tilde{W}$ is a $d$-dimensional Brownian motion, for $d \geq 1, \mu^{X}$ is a random measure of the jumps of $X$, $v(\mathrm{~d} t, \mathrm{~d} z)=\ell_{t}(\mathrm{~d} z) \mathrm{d} t$ is the compensator of the jump measure $\mu^{X}$ which we assume to be deterministic, $\omega:[0, T] \times \mathbb{R}^{d} \rightarrow \mathbb{R}^{d}, \sigma:[0, T] \times \mathbb{R}^{d} \rightarrow \mathbb{R}^{d \times d}$ are such that $\varrho(t, x)=\sigma(t, x) \sigma(t, x)^{\top}$, for $t \in[0, T]$ and $x \in \mathbb{R}^{d}$. Moreover, $\iota(t, z) \in \mathbb{R}^{d}$, for $t \in[0, T]$ and $z \in \mathbb{R}$ and is such that $\int_{\mathbb{R}} \iota^{\top}(t, z) \iota(t, z) \ell_{t}(\mathrm{~d} z)<\infty$, for all $t \in[0, T]$.

Moreover, assume the following affine structure for the time-dependent parameters of the SDE for $X$

$$
\varrho(t, x)=\varrho(t)+\sum_{i=1}^{d} x_{i} \alpha_{i}(t)
$$




$$
\omega(t, x)=\omega(t)+\sum_{i=1}^{d} x_{i} \beta_{i}(t)
$$

where $\varrho(t)$ and $\alpha_{i}(t)$ are $d \times d$ matrices and $\omega(t)$ and $\beta_{i}(t)$ are $d$-vectors. Let

$$
\alpha(t)=\left(\alpha_{1}(t), \ldots, \alpha_{d}(t)\right), \quad \beta(t)=\left(\beta_{1}(t), \ldots, \beta_{d}(t)\right)
$$

We consider a real-valued process $R$ for which we impose the following affine structure

$$
R(t)=c+\gamma^{\top} X(t)
$$

for $c \in \mathbb{R}$ and $\gamma \in \mathbb{R}^{d}$. We introduce some conditions on the parameters $\left(\varrho(t), \alpha(t), \omega(t), \beta(t), c, \gamma, \ell_{t}\right)$, $t \in[0, T]$ in the following definition.

Definition A1. The parameters $\left(\varrho(t), \alpha(t), \omega(t), \beta(t), c, \gamma, \ell_{t}\right), t \in[0, T]$, are called strongly admissible if the following conditions hold

- $\varrho(t), \alpha_{i}(t) \in \operatorname{Sem}^{d}$, for $i=1, \ldots, d$.

- $\left(\varrho(t), \alpha(t), \omega(t), \beta(t), \ell_{t}\right)$, are continuous in $t \in[0, T]$,

.

$$
\begin{aligned}
& z \mathbf{1}_{|z| \leq 1}\left(\iota^{k}(t, z)\right) \ell_{t}(\mathrm{~d} z) \text { is weakly continuous on } \mathbb{R}, \text { for } 0 \leq k \leq d, \\
& z^{2} \mathbf{1}_{|z| \leq 1}\left(\iota^{k}(t, z)\right) \ell_{t}(\mathrm{~d} z) \text { is weakly continuous on } \mathbb{R}, \text { for } 0 \leq k \leq d,
\end{aligned}
$$

We compute in the following theorem the discounted moment generating function of $X(T)$, conditional on the information at time $t \leq T$ in terms of the solution to the Riccati equations (A5). For a proof we refer to (Kallsen and Muhle-Karbe 2010, Theorems 2.6 and 5.1).

Theorem A1. Assume the parameters $\left(\varrho(t), \alpha(t), \omega(t), \beta(t), c, \gamma, \ell_{t}\right), t \in[0, T]$, are strongly admissible parameters. Let $u \in \mathbb{C}^{d}$ and $(\phi(\cdot, T, u), \psi(\cdot, T, u)):[0, T] \rightarrow \mathbb{C} \times \mathbb{C}^{d}$ be $C^{1}$-functions satisfying

1. $\int_{\{|z|>1\}} \mathrm{e}^{\psi(t, T, u)^{\top} \iota(t, z)} \ell_{t}(\mathrm{~d} z)<\infty, \quad \forall t \in[0, T], \quad u \in \mathbb{C}^{d}$,

2.

$$
\begin{aligned}
\partial_{t} \phi(t, T, u)=- & \psi(t, T, u)^{\top} \omega(t)-\frac{1}{2} \psi(t, T, u)^{\top} \varrho(t) \psi(t, T, u) \\
& -\int_{\mathbb{R}}\left[e^{\psi(t, T, u)^{\top} \iota(t, z)}-1-\psi(t, T, u)^{\top} \iota(t, z)\right] \ell_{t}(\mathrm{~d} z)+c, \\
\partial_{t} \psi_{i}(t, T, u) & =-\beta_{i}(t)^{\top} \psi(t, T, u)-\frac{1}{2} \psi(t, T, u)^{\top} \alpha_{i}(t) \psi(t, T, u)+\gamma_{i}, \quad 1 \leq i \leq d, \\
\phi(T, T, u) & =0, \\
\psi(T, T, u) & =u .
\end{aligned}
$$

Then we have

$$
\mathbb{E}\left[\mathrm{e}^{-\int_{t}^{T} R(s) \mathrm{d} s} \mathrm{e}^{u^{\top} X(T)} \mid \mathcal{F}_{t}\right]=\mathrm{e}^{\phi(t, T, u)+\psi(t, T, u)^{\top} X(t)}, \quad t \leq T .
$$

This latter result allows the use of Fourier transform techniques to compute derivative prices written on affine models. 


\section{Appendix B. Proof of Lemma 2}

Inserting (3) and (1) in (33), we find after some rearrangement

$$
\mathrm{d} r(t)+B_{2}(t) r(t) \mathrm{d} t=\left[A(t)-B_{1}(t) \chi(t) b\right] \mathrm{d} t+B_{1}(t) \mathrm{d} \xi(t)-B_{1}(t) \int_{0}^{t} M(t-u) \xi(u) \mathrm{d} u \mathrm{~d} t .
$$

Multiplying both sides of (A6) with $\mathrm{e}^{\int_{0}^{t} B_{2}(s) \mathrm{d} s}$, applying the product rule to $\mathrm{d}\left(\mathrm{e}^{-\int_{0}^{t} B_{2}(u) \mathrm{d} u} B_{1}(t) \xi(t)\right)$, integrating both sides from zero to $t$, and dividing by $\mathrm{e}_{0}^{t} B_{2}(u) \mathrm{d} u$ we get

$$
\begin{aligned}
r(t)= & \mathrm{e}^{-\int_{0}^{t} B_{2}(u) \mathrm{d} u} r(0)+\int_{0}^{t} \mathrm{e}^{-\int_{s}^{t} B_{2}(u) \mathrm{d} u}\left[A(s)-B_{1}(s) \chi(s) b\right] \mathrm{d} s \\
& +B_{1}(t) \xi(t)-\mathrm{e}^{-\int_{0}^{t} B_{2}(u) \mathrm{d} u} B_{1}(0) \xi_{0}-\int_{0}^{t} \mathrm{e}^{-\int_{s}^{t} B_{2}(u) \mathrm{d} u} B_{1}(s) B_{2}(s) \xi(s) \mathrm{d} s \\
& -\int_{0}^{t} \mathrm{e}^{-\int_{s}^{t} B_{2}(u) \mathrm{d} u} \xi(s) \mathrm{d} B_{1}(s)-\int_{0}^{t} \mathrm{e}^{-\int_{s}^{t} B_{2}(u) \mathrm{d} u} B_{1}(s) \int_{0}^{s} M(s-u) \xi(u) \mathrm{d} u \mathrm{~d} s .
\end{aligned}
$$

Applying the triangle inequality, the boundedness of $\chi, A, B_{1}$ and $B_{2}$ and the assumption on $\xi_{0}$ we bound $r(t)$ for $t \leq T$ by

$$
\begin{aligned}
|r(t)| \leq & \mathrm{e}^{K_{1} T}\left(K_{2}+K_{3} T\right)+K_{4}|\xi(t)|+\mathrm{e}^{K_{1} T} K_{5} \int_{0}^{t}|\xi(s)| \mathrm{d} s \\
& +\mathrm{e}^{K_{1} T} \int_{0}^{t}|\xi(s)|\left|\mathrm{d} B_{1}(s)\right|+\mathrm{e}^{K_{1} T} K_{4} \int_{0}^{t} \int_{0}^{s}|M(s-u)||\xi(u)| \mathrm{d} u \mathrm{~d} s,
\end{aligned}
$$

for some positive constants $K_{1}, \ldots, K_{5}$. Because $B_{1}$ is of bounded variation on $[0, T]$, we further obtain

$$
|r(t)| \leq \mathrm{e}^{K_{1} T}\left(K_{2}+K_{3} T\right)+\mathrm{e}^{K_{1} T} K_{6}\left(T+\int_{0}^{t} \int_{0}^{s}|M(s-u)| \mathrm{d} u \mathrm{~d} s\right) \sup _{0 \leq s \leq t}|\xi(s)|,
$$

with some additional positive constant $K_{6}$. By Cauchy-Schwartz inequality,

$$
\begin{aligned}
\int_{0}^{T} \int_{0}^{s}|M(s-u)| \mathrm{d} u \mathrm{~d} s & \leq \int_{0}^{T}\left(\int_{0}^{s} 1 \mathrm{~d} s\right)^{1 / 2}\left(\int_{0}^{s}|M(s-u)|^{2} \mathrm{~d} u\right)^{1 / 2} \mathrm{~d} s \\
& \leq\left(\int_{0}^{T}|M(u)|^{2} \mathrm{~d} u\right)^{1 / 2} \int_{0}^{T} s^{1 / 2} \mathrm{~d} s=\frac{2}{3} T^{3 / 2}\left(\int_{0}^{T} M^{2}(u) \mathrm{d} u\right)^{1 / 2}
\end{aligned}
$$

which is finite by assumption. Hence, we find,

$$
|r(t)| \leq \tilde{K}_{1}+\tilde{K}_{2} \sup _{0 \leq s \leq t}|\xi(s)|,
$$

where $\tilde{K}_{1}, \tilde{K}_{2}$ are two positive constants, depending on $T$. As $\sqrt{ } \cdot$ is an increasing function,

$$
\sup _{0 \leq s \leq t}|\xi(s)|=\sup _{0 \leq s \leq t} \sqrt{|\xi(s)|^{2}} \leq \sqrt{\sup _{0 \leq s \leq t}|\xi(s)|^{2}}
$$


and therefore, by an elementary inequality,

$$
|r(t)|^{2} \leq C_{1}+C_{2} \sup _{0 \leq s \leq t}|\xi(s)|^{2},
$$

for some positive constants $C_{1}, C_{2}$ (depending on $T$ ).

Analogous to (33) we can transform (34) into

$$
\mathrm{d} \rho(t)+\bar{B}_{3}(t) \rho(t) \mathrm{d} t=\left[\bar{A}(t)-\bar{B}_{1}(t) \chi(t) b-\bar{B}_{2}(t) r(t)\right] \mathrm{d} t+\bar{B}_{1}(t) \mathrm{d} \xi(t),
$$

Multiplying both sides of (A9) with $\mathrm{e}^{\int_{0}^{t} \bar{B}_{3}(s) \mathrm{d} s}$, applying the product rule to $\mathrm{d}\left(\mathrm{e}^{-\int_{0}^{t} \bar{B}_{3}(u) \mathrm{d} u} \bar{B}_{1}(t) \xi(t)\right)$, integrating both sides from zero to $t$, and dividing by $\mathrm{e}^{\int_{0}^{t} \bar{B}_{3}(u) \mathrm{d} u}$, we get

$$
\begin{aligned}
\rho(t)= & \mathrm{e}^{-\int_{0}^{t} \bar{B}_{3}(u) \mathrm{d} u} \rho(0)+\int_{0}^{t} \mathrm{e}^{-\int_{s}^{t} \bar{B}_{3}(u) \mathrm{d} u}\left[\bar{A}(s)-\bar{B}_{1}(s) \chi(s) b-\bar{B}_{2}(s) r(s)\right] \mathrm{d} s \\
& +\bar{B}_{1}(t) \xi(t)-\mathrm{e}^{-\int_{0}^{t} \bar{B}_{3}(u) \mathrm{d} u} \bar{B}_{1}(0) \xi_{0}-\int_{0}^{t} \mathrm{e}^{-\int_{s}^{t} \bar{B}_{3}(u) \mathrm{d} u} \bar{B}_{1}(s) \bar{B}_{3}(s) \xi(s) \mathrm{d} s \\
& -\int_{0}^{t} \mathrm{e}^{-\int_{s}^{t} \bar{B}_{3}(u) \mathrm{d} u} \xi(s) \mathrm{d} \bar{B}_{1}(s) .
\end{aligned}
$$

Taking absolute values and appealing to the boundedness assumptions along with the estimate (A8) we can bound $\rho(t)$ in (A10) for $t \leq T$ by the same arguments as for $r$ to arrive at

$$
|\rho(t)|^{2} \leq C_{3}+C_{4} \sup _{0 \leq s \leq t} \xi^{2}(s),
$$

for positive constants $C_{3}$ and $C_{4}$ (depending on $T$ ). The result follows.

\section{References}

Abi Jaber, Eduardo, Martin Larsson, and Sergio Pulido. 2019. Affine Volterra processes. The Annals of Applied Probability 29: 3155-3200. [CrossRef]

Barndorff-Nielsen, Ole E., Fred Espen Benth, and Almut E.D. Veraart. 2011. Ambit processes and stochastic partial differential equations. In Advanced Mathematical Methods for Finance. G. Di Nunno and B. Øksendal, Eds. Heidelberg: Springer, pp. 35-74.

Barndorff-Nielsen, Ole E., Fred Espen Benth, and Almut E.D. Veraart. 2013. Modelling energy spot prices by volatility modulated Lévy-driven Volterra processes. Bernoulli 19: 803-845. [CrossRef]

Barndorff-Nielsen, Ole E., Fred Espen Benth, and Almut E.D. Veraart. 2014. Modelling electricity futures by ambit fields. Advances in Applied Probability 46: 719-745. [CrossRef]

Barndorff-Nielsen, Ole E., and Jürgen Schmiegel. 2009. Brownian semistationary processes and volatility/intermittency. In Advanced Financial Modeling. H. Albrecher, W. Runggaldier and W. Schachermayer, Eds. Radon Series on Computational and Applied Mathematics 8. Berlin: W. de Gruyter, pp. 1-26.

Belbas, S.A. 2007. A new method for optimal control of Volterra integral equations. Applied Mathematics and Computation 189: 1902-1915. [CrossRef]

Espen, Benth Fred, and Saltyte-benth Jurate. 2012. Modeling and Pricing in Financial Markets for Weather Derivatives. Advanced Series on Statistical Science and Applied Probability. Singapore: World Scientific, vol. 17.

Espen, Benth Fred, Saltyte-benth Jurate, and Steen Koekebakker. 2008. Stochastic Modeling of Electricity and Related Markets. Advanced Series on Statistical Science and Applied Probability. Singapore: World Scientific, vol. 11.

Espen, Benth Fred, Claudia Klüppelberg, Gernot Müller, and Linda Vos. 2014. Futures pricing in electricity markets based on stable CARMA spot models. Energy Economics 44: 392-406. 
Björk, Tomas. 2009. Arbitrage Theory in Continuous Time, 3rd ed. Oxford Finance Series. Oxford: Oxford University Press. Comte, Fabio, and Eric Renault. 1996. Long memory continuous time models. Journal of Econometrics 73: 101-149. [CrossRef]

Cont, Rama. 2001. Empirical properties of asset returns: Stylized facts and statistical issues. Quantitative Finance 1: 223-236. [CrossRef]

Cuchiero, Christa, and Josef Teichmann. 2018. Generalized Feller processes and Markovian lifts of stochastic Volterra processes: The affine case. arXiv arXiv:1804.10450.

Ding, Zhuanxin, Clive W.J. Granger, and Robert F. Engle. 1993. A long memory property of stock market returns and a new model. Journal of Empirical Finance 1: 83-106. [CrossRef]

Duan, Jin-Chuan, and Stanley R. Pliska. 2004. Option valuation with co-integrated asset prices. Journal of Economic Dynamics and Control 28: 727-754. [CrossRef]

Duffie, Darrell, Damir Filipović, and Walter Schachermayer. 2003. Affine processes and applications in finance. The Annals of Applied Probability 13: 984-1053.

Duffie, Darrell, Jun Pan, and Kenneth Singleton. 2000. Transform analysis and asset pricing for affine jump-diffusions. Econometrica 68: 1343-1376. [CrossRef]

El Euch, Omar, and Mathieu Rosenbaum. 2018. Perfect hedging in rough Heston models. The Annals of Applied Probability 28: 3813-3856. [CrossRef]

Eydeland, Alexander, and Krzysztof Wolyniec. 2003. Energy and Power Risk Management: New Developments in Energy Modeling, Pricing and Hedging. Hoboken: Wiley Finance.

Filipović, Damir. 2009. Term Structure Models. A Graduate Course, 1st ed. Berlin/Heidelberg: Springer.

Gatheral, Jim, Thibault Jaisson, and Mathieu Rosenbaum. 2018. Volatility is rough. Quantitative Finance 18: 933-949. doi:10.1080/14697688.2017.1393551. [CrossRef]

Geman, Hélyette. 2005. Commodities and Commodity Derivatives. Hoboken: Wiley Finance.

Granger, Clive W.J., and Zhuanxin Ding. 1999. Stylized Facts on the Temporal and Distributional Properties of Daily Data From Speculative Markets. UCSD Department of Economics Discussion Paper. San Diego: UCSD Department of Economics, pp. 94-19.

Gripenberg, Gustaf, Stig-Olof Londen, and Olof Staffans. 1990. Volterra Integral and Functional Equations. Cambridge: Cambridge University Press, vol. 34.

Holden, Helge, Bernt Øksendal, Jan Ubøe, and Tusheng Zhang. 2010. Stochastic Partial Differential Equations. Berlin: Springer.

Kallsen, Jan, and Johannes Muhle-Karbe. 2010. Exponentially affine martingales, affine measure changes and exponential moments of affine processes. Stochastic Processes and their Applications 120: 163-181. [CrossRef]

Keller-Ressel, Martin, and Eberhard Mayerhofer. 2015. Exponential moments of affine processes. The Annals of Applied Probability 25: 151-168. [CrossRef]

Klebaner, Fima, and Robert Liptser. 2014. When a stochastic exponential is a true martingale. Extension of the Beneš method. Theory of Probability \& Its Applications 58: 38-62.

Lipster, Robert Shevilevich, and Albert Nikolaevich Shiriaev. 1984. Statistics of Random Processes I, General Theory, 2nd ed. Berlin: Springer.

Øksendal, Bernt, and Agnes Sulem 2009. Applied Stochastic Control of Jump Diffusions, 3rd ed. Berlin: Springer.

Øksendal, Bernt, and Tusheng Zhang. 2010. Optimal control with partial information for stochastic Volterra equations. International Journal of Stochastic Analysis 2010: 329185. [CrossRef]

Ortiz-Latorre, Salvador. 2017. A new pricing measure in the Barndorff-Nielsen-Shephard model for commodity markets. In Extended Abstracts Summer 2015. Berlin: Springer, pp. 133-139.

Paschke, Raphael, and Marcel Prokopczuk. 2010. Commodity derivatives valuation with autoregressive and moving average components in the price dynamics. Journal of Banking and Finance 34: 2742-2752. [CrossRef]

Protter, Philip. 1985. Volterra equations driven by semimartingales. The Annals of Probability 13: 519-530. [CrossRef]

Protter, Philip. 2004. Stochastic Integration and Differential Equations: Version 2.1. Applications of Mathematics. Berlin: Springer.

Samuelson, Paul A. 2016. Proof that properly anticipated prices fluctuate randomly. In The World Scientific Handbook of Futures Markets. Singapore: World Scientific, pp. 25-38.

Schwartz, Eduardo, and James E. Smith. 2000. Short-term variations and long-term dynamics in commodity prices. Management Science 46: 893-911. [CrossRef] 
Takahashi, Masafumi. 1996. Non-ideal Brownian motion, generalised Langevin equation and its application to the security market. Financial Engineering and the Japaneses Markets 3: 87-119. [CrossRef]

Veraar, Mark. 2012. The stochastic Fubini theorem revisited. Stochastics 84: 543-551. [CrossRef]

(C) 2020 by the authors. Licensee MDPI, Basel, Switzerland. This article is an open access article distributed under the terms and conditions of the Creative Commons Attribution (CC BY) license (http://creativecommons.org/licenses/by/4.0/). 